\title{
Jungpleistozäne und altholozäne Faunen (Gastropoda und Mammalia) vom Euerwanger Bühl bei Greding (Fränkischer Jura)
}

\author{
Von Wighart v. Koenigswald und Wolfgang Rähle, Tübingen
}

Mit 9 Abbildungen und 7 Tabellen

$\mathrm{Z}$ u s a m m e n f a s s u n g. Aus einer Höhlenruine werden Kleinsäuger- und Schneckenfaunen qualitativ und quantitativ erfaßt. Ein Komplex gehört in das Würmglazial, die übrigen in das Altholozän, und zwar nach den C14-Datierungen in das Präboreal und frühe Boreal. Altholozäne Kleinsäugerfaunen sind in Süddeutschland durch Pityms subterraneus sowie pleistozäne Reliktformen charakterisiert. In der Gastropodenfauna wird Zebrina detrita in einer reinen Discus ruderatus / Nesovitrea petronella Fauna nachgewiesen.

A bstract. Small mammals and gastropod faunas out of a collapsed cave were quantitatively and qualitatively studied. One complex belongs to the last glacial, the others to the early holocene, or precicely Preboreal and early Boreal according to C14 age dating. Early holocene small mammal faunas in southern Germany are represented by Pitymys subterraneus as well as pleistocene relict forms. The gastropod fauna contains Zebrina detrita in a typical Discus ruderatus / Nesovitrea petronella fauna.

\section{Einleitung}

In zahlreichen fränkischen Höhlen kann man dicht unter der Oberfläche eine Vermischung pleistozäner und holozäner Faunenelemente feststellen. Zeitlich eng begrenzte und daher unvermischte Faunen aus dem Übergangsbereich Pleistozän/Holozän sind bisher aus diesem Gebiet kaum bekannt. Am Euerwanger Bühl bei Greding war es nun möglich, einen detailierten Eindruck von solchen Faunen zu gewinnen, da hier in eng begrenzten Komplexen Kleinsäugerfaunen gemeinsam mit reichhaltigen Schneckenfaunen gefunden wurden.

Herrn Ministerialrat a. D. Alfred Forstmeyer sei an dieser Stelle noch einmal herzlich dafür gedankt, daß er die Fundbergung mit so großem persönlichem und dabei äußerst umsichtigen Einsatz durchgeführt hat und auch dafür, daß er das von ihm zusammengetragene Material sowie seine Unterlagen so großzügig einer Bearbeitung zur Verfügung gestellt hat. Herrn Dr. W. Taute (Tübingen) verdanken wir die Klassifizierung der wenigen Artefakte und die archäologische Einstufung dieser Funde. Ebenso danken wir dem C14-Labor des Instituts für Umweltphysik der Universität Heidelberg für die Durchführung der absoluten Altersbestimmungen. Unser Dank gilt auch Herrn Markert (Tübingen) für die Bestimmung der Reptilienreste (MARKERT 1975) sowie Herrn Dr. F. Schweingruber (Birmensdorf, Schweiz) für die der Holzkohlenfunde.

Diese Studie entstand im Rahmen des Sonderforschungsbereiches 53, „Paläontologie, mit besonderer Berücksichtigung der Palökologie“, an der Universität Tübingen und wird als "FossilVergesellschaftungen Nr. 33“ geführt.

\section{Die Fundsituation}

Wo der Fränkische Jura aus einer NE-SW-Richtung in die N-S-Richtung umbiegt, durchschneidet das Schwarzachtal bei Greding die Albtafel. Westlich dieses Tales dehnt sich eine tertiäre Verebnungsfläche im Niveau des Weiß-Jura- $\gamma$ aus, die von dolomitisierten Riffkomplexen um 60-70 m überragt wird. Einer dieser Härtlinge ist der Euerwanger Bühl. Er liegt etwa $3 \mathrm{~km}$ südlich der Autobahnausfahrt Greding. Am Südhang dieses Ber- 
ges wurde beim Abbau von „Bergkies“ ein verstürztes Höhlensystem freigelegt. (MTB

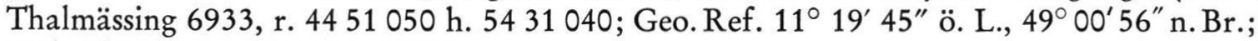
Höhenlage: etwa 470 über NN.)

Ministerialrat a. D. Alfred Forstmeyer aus Greding konnte hier während des Abbaus und vor allem durch eigene Grabungen sowohl ein umfangreiches Faunenmaterial des Pleistozäns und des Altholozäns, als auch einige mesolithische Steinwerkzeuge bergen. Besonders ist dabei hervorzuheben, daß die Funde in Komplexen nach unterschiedlicher horizontaler und vertikaler Position geborgen und getrennt gehalten worden sind, was eine lohnende Bearbeitung möglich machte. Die Komplexe dokumentieren die ökologischen Verhältnisse während verschiedener Stadien der Verfüllung der Höhlenruine. Die einzelnen Teile der Höhle sind zu unterschiedlicher Zeit eingestürzt. Die entstandenen oberflächlichen Hohlformen wurden von oben her mit scharfkantigem hangabwärtskriechendem Dolomitschutt verfüllt. Der Hauptkessel wurde im Pleistozän unter kaltzeitlichen Bedingungen verfüllt, wie die Fauna zeigt. Dem spärlichen Zwischenmittel aus Dolomitsand fehlt jede Humuskomponente. Der nach Nordosten abgehende Höhlenarm „Schwarze Wand" ist wahrscheinlich erst unmittelbar vor Beginn des Holozäns eingestürzt. Die Füllung besteht auch in diesem Teil aus Dolomitschutt, dessen Zwischenmittel allerdings stark humos ist, was zusammen mit den zahlreichen Holzkohleeinlagerungen eine dunkle Sedimentfarbe ergibt. Auf diesen Farbunterschied bezieht sich die Benennung dieses Höhlenteils als "Schwarze Wand“, da er bei der Gewinnung des „Bergkieses“ als dunkle Abbauwand in Erscheinung trat.

Bei den Faunen aus dem Euerwanger Bühl sind zwei $\mathrm{Ha}$ u p tg r u p pen zu unterscheiden: Die e rs te umfaßt eine Großsäugerfauna mit deutlich kaltzeitlichem Charakter aus den tieferen Teilen des mit „Bergkies“ verfüllten Höhlensystems.

Vertreten sind folgende Arten:

$\begin{array}{ll}\text { Mammonteus primigenius } & \text { Mammut } \\ \text { Equus remagensis }(=\text { germanicus } \text { praeocc. }) & \text { Pferd } \\ \text { Rangifer tarandus } & \text { Rentier } \\ \text { Bos primigenius } & \text { Ur } \\ \text { Bison priscus } & \text { Wisent }\end{array}$

Die Bearbeitung dieser Fauna ist durch Prof. Dr. F. Heller, Erlangen, vorgesehen, der einen besonders gut erhaltenen Pferdeunterkiefer aus dieser Fauna bereits in einem anderen Zusammenhang publiziert hat (Heller 1973).

Die $\mathrm{z}$ we it e Hauptgruppe, die Gegenstand dieser Arbeit ist, besteht aus mehreren Komplexen umfangreicher Kleinfauna (Schnecken und Kleinsäuger), mit nur relativ wenig Großsäugerresten. Diese Fundkomplexe stammen aus dem Würm und besonders dem Altholozän. Hier werden vor allem die Kleinsäugerreste (durch v. K.) und die Gastropoden (durch R.) beschrieben. Diese Untersuchungen sind ein Teil einer umfassenden Studie über die Faunenveränderung an der Pleistozän-Holozän-Grenze. Die Holzkohlenreste aus den Komplexen A2, A1 und R wurden von F. SCHwEINGRUBER, Eidgenössische Anstalt für das forstliche Versuchswesen, Birmensdorf (Schweiz), nach Holzarten untersucht und die Ergebnisse, die auch an anderer Stelle publiziert werden, sind hier einbezogen. Schon die Holzkohlen könnten Zeugnis der Anwesenheit des frühen Menschen am Euerwanger Bühl sein. Durch Feuersteinabschläge und einige ausgearbeitete Geräte ist menschliche Aktivität mit Sicherheit in den Komplexen C, A2, R VI-II belegt. Die Artefakte lassen sich nach W. TAute, Tübingen, am ehesten zum Beuronien A/B, das heißt dem frühen Mesolithikum, stellen. Diese Kulturstufe wird von TAUTE (1971) in das Präboreal und frühe Boreal gestellt; damit stehen auch die $\mathrm{C}^{14}$-Datierungen vom Euerwanger Bühl in gutem Einklang, die Herr Forstmeyer im 2. Physikalischen Institut der Universität Heidelberg an Holzkohle durchführen ließ (s. Abb. 8). 


\section{Die Fundkomplexe}

Die Fundkomplexe H, F, E, D, C, B, A2, A1 und R, in denen die hier behandelten Faunen der 2. Hauptgruppe gefunden werden, stammen nicht aus einem Profil, sondern sind räumlich auf den Nord- und Ostteil der Höhlenruine verteilt (Abb. 1). Die Altersabfolge dieser Komplexe ergibt sich teilweise durch die C14-Datierungen und teilweise aus der Superposition. $\mathrm{H}$ wird aus faunistischen Gründen älter als $\mathrm{F}$ eingestuft und ist der älteste hier behandelte Komplex. R entspricht zwar in der Höhenlage etwa A, entspricht aber in der Faunenzusammensetzung nur A2 und tieferen Horizonten.

Kleinsäugeranreicherungen in Höhlen oder an Felsen, wie sie am Euerwangerbühl gegeben sind, gehen in der Regel auf Gewölle von Eulenvögeln zurück. Da der Flugbereich einen Umkreis von 5-10 km abdeckt, sind in der Fauna auch die Biotope dieses Gebietes summiert. Gerade durch diese Verwischung von kleinräumigen ökologischen Unterschieden gewinnen diese Gewöllefaunen erheblich an stratigraphischer Aussagekraft.

Bei der quantitativen Auswertung der Kleinsäugerfaunen werden die Mindestzahlen der belegten Individuen-Hälf $\mathrm{t}$ e $\mathrm{n}$ angegeben. Das entspricht der Auszählung der $\mathrm{M}_{1}$ bei Microtinen und eliminiert die Zufälligkeiten der Erhaltung von rechten und linken
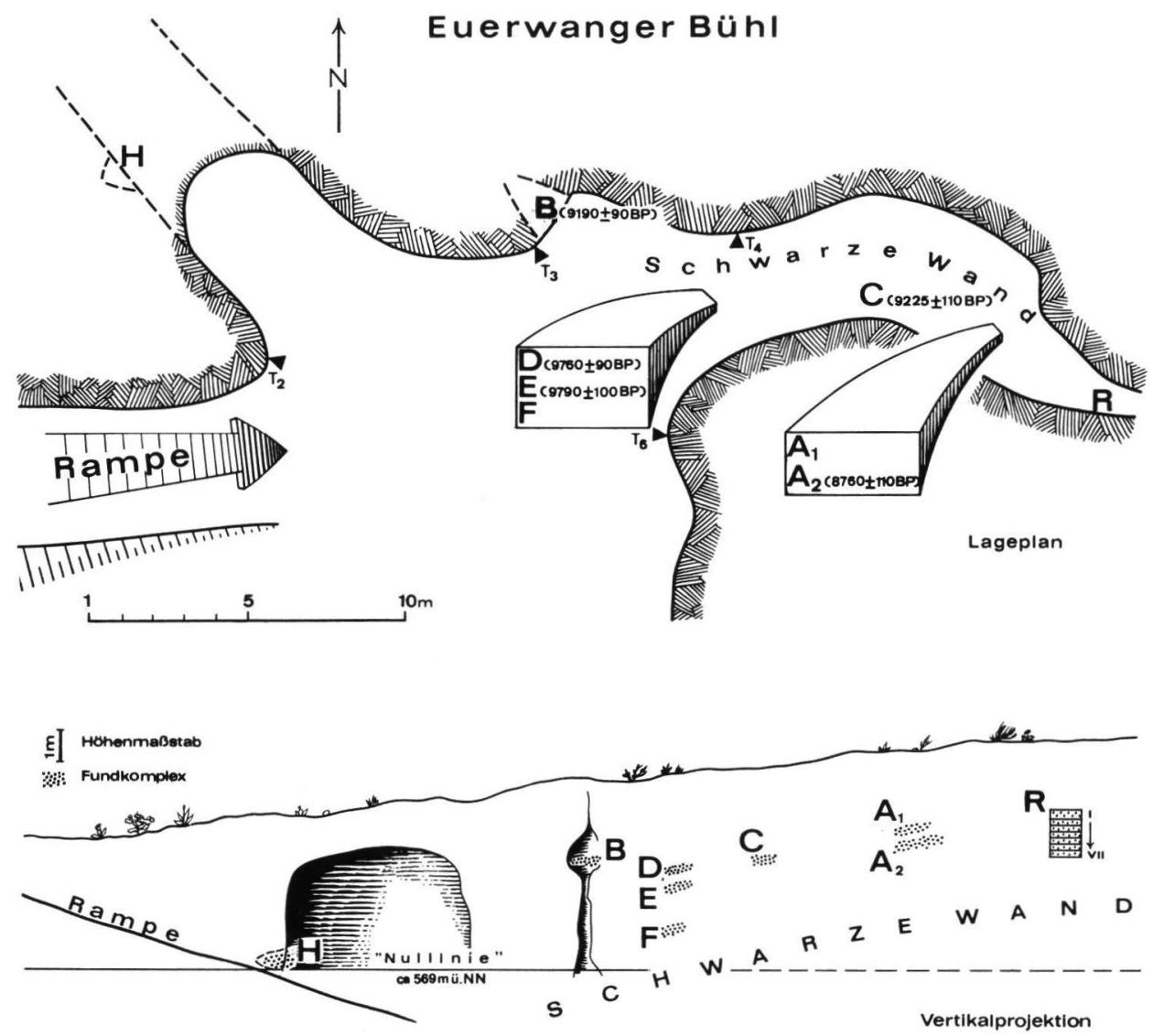

Abb. 1. Euerwanger Bühl bei Greding. Lage der Fundkomplexe in der Planskizze und der Vertikalprojektion der Höhlenruine. 
Körperteilen. Auf diese Weise wird besonders bei geringeren Individuenzahlen ein besserer Vergleichswert gewonnen. Quantitativ untereinander vergleichbar sind bei den Aufsammlungen vom Euerwanger-Bühl nur die mittelgroßen Nager, Clethrionomys, Microtus, Pitymys und Apodemus, da deren Kiefer ungefähr gleich groß sind und so nahezu die gleiche Chance hatten, gefunden zu werden, während die deutlich größeren Kiefer von Arvicola bei der Aufsammlung sicher bevorzugt und die kleineren Kiefer, etwa die von Sicista, leichter übersehen wurden.

Auch für die Mollusken wurden auf Grund der in den Proben vorhandenen Gehäusefragmente versucht, die Mindestzahlen der nachzuweisenden Individuen zu bestimmen. Vergleichbare Werte ergaben sich jedoch nur innerhalb der einzelnen Fundkomplexe, da das Material auf verschiedene Weise gewonnen wurde. So sind in Fundkomplex A die Kleinschnecken deutlich unterrepräsentiert, da hier die Proben nur oberflächlich abgesammelt wurden. In Komplex R, wo das Material geschlämmt worden ist, erreichen diese Formen einen viel größeren Anteil.

Bei der "malakozoologischen" Analyse werden für die einzelnen Schichten in Anlehnung an LOžEK (1964) Molluskenspektren aufgestellt. Dabei werden die einzelnen Arten - wie in der Artenliste (Tab. 6) - nach ihren ökologischen Ansprüchen gruppiert und die Anteile dieser ökologischen Gruppen an der Gesamtfauna prozentual ausgewiesen. In den Molluskenspektren der Arten bildet die jeweilige Gesamtzahl der Arten die Basis, in den Molluskenspektren der Individuen die Gesamtindividuenzahl aus der betreffenden Schicht (Abb. 3 u. 4).

Danebengestellt werden Molluskenspektren der Arten bzw. Individuen, bei denen die Mollusken nach ihrer biostratigraphischen Bedeutung in Gruppen zusammengefaßt worden sind (Abb. 5 u. 6). Die Zuordnung zu den drei in Frage kommenden Gruppen ist der Artenliste zu entnehmen, wo dafür folgende Signaturen zur Verwendung kommen:

! : Arten, die für feucht-warme Klimaabschnitte bezeichnend sind.

* : Arten, die für trockene und warme Klimaabschnitte bezeichnend sind (pontische und mediterrane Arten).

○ : Arten, die klimatischen Gegebenheiten gegenüber mehr oder weniger indifferent sind.

\section{Komplex H}

In der nördlichen Seitenhöhle zwischen $T_{2}$ und $T_{3}$ fand sich in einer Nische der westlichen Wand, etwa in der Höhenlage der von Herrn Forstmeyer angelegten „Nullinie“, eine umfangreiche Knochenanreicherung, die ausschließlich von Kleinsäugern stammte. Schnecken sind in diesem Komplex nicht überliefert. Die Knochen lagen zum Teil an der Oberfläche, zum Teil im liegenden Dolomitsand. Die Farbe der Knochen ist grau und ähnelt der der pleistozänen Komplexe der ersten Hauptgruppe.

Die Kleinsäugerreste dürften aus Gewöllen stammen. Es muß sich um einen großen Eulenvogel gehandelt haben, da mehrere Arvicola-Schädel unzerdrückt erhalten sind.

In der Fauna, die vorwiegend aus Nagetieren besteht, fehlen auffallenderweise die Insektivoren. Diese beiden Besonderheiten lassen den Uhu (Bubo bubo) und die Sumpfohreule (Asio flammeus) als Verursacher dieser Gewölleanreicherung in Frage kommen. JáNOssy (1970) weist darauf hin, daß Arvicola in Uhugewöllen häufiger ist als in denen anderer Eulen. Prof. E. von Lehmann, Bonn, verdanken wir den Hinweis, daß auch in den Gewöllen der Sumpfohreule Arvicola reichlich und nach seinen Beobachtungen mit völlig intakten Schädeln erhalten ist (s. a. KumERLOeve 1968). 
Tabelle 1

Euerwanger Bühl. Faunenzusammensetzung im Komplex $\mathrm{H}$.

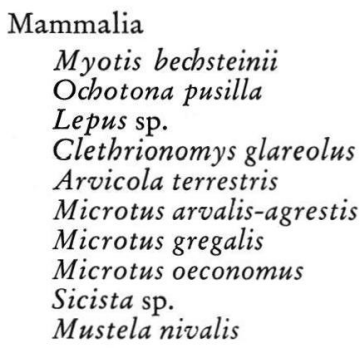

$\begin{array}{rc}4 & \text { Individuenhälften } \\ 4 & \\ 1 & \\ 6 & 3 \% \\ 30 & \\ 103 & 58 \% \\ 40 & 23 \% \\ 29 & 16 \% \\ 1 & \\ 2 & \end{array}$

$100 \%(\mathrm{n}=178)$

Der Artbestand umfaßt hauptsächlich Nagetiere. Am häufigsten sind Reste der Microtus arvalis-agrestis-Gruppe, die zum überwiegenden Teil aus Microtus arvalis besteht. Das läßt sich daraus schließen, daß von den beiden für M. agrestis typischen Unterscheidungsmerkmalen, nämlich der Ausbildung eines zweiten Innenprismas am $\mathrm{M}^{2}$ sowie der Lage des Foramen mandibulae mitten auf dem Knochenwulst (GAFFrey 1952), nur das erste in wenigen Ausnahmefällen beobachtet werden konnte. Wenn bei beiden Merkmalen auch Ausnahmen vorkommen, so liegt doch zumindest ein erhebliches Übergewicht von Microtus arvalis vor. In der Häufigkeit folgen die übrigen Wühlmäuse: Microtus gregalis, M. oeconomus, Arvicola und sehr untergeordnet Clethrionomys.

Die Fauna aus Komplex $\mathrm{H}$ kann als kaltzeitlich angesprochen werden, da Microtus gregalis mit einem so hohen Prozentsatz und Arvicola in der für die letzte Kaltzeit typischen Großform vorliegen (vgl. S. 161). Dieses Bild bekräftigen die Belege von Sicista und Ochotona sowie der Anteil von Microtus oeconomus von mehr als 10\%.

Diese Art kommt zwar ebenso wie Sicista als Reliktform auch noch im Postglazial vor, erreicht dort aber nur einen wesentlich geringeren Anteil (z. B. Burghöhle Dietfurt 12 a: weniger als $2 \%$ ). Umgekehrt verhält sich der quantitative Anteil von Clethrionomys. Diese Art tritt zwar in geeigneten Biotopen mit geringen Individuenzahlen auch während der Kaltzeiten auf, wird aber erst mit der postglazialen Klimaverbesserung zusammen mit Apodemus häufig und damit zur Leitform. Diese kaltzeitliche Fauna stammt aus einem relativ feuchten Biotop, wie der hohe Anteil von Arvicola und Microtus oeconomus vermuten läßt. Eine stratigraphische Einstufung wird auf S. 175 gegeben.

\section{Komplex F}

Als „Schwarze Wand“ wird, wie schon erwähnt, die Verfüllung eines ehemaligen Höhlenarmes bezeichnet, der vom Hauptraum nach Nord-Osten abgeht. Die Bezeichnung bezieht sich auf die starke Humusinfiltration der Füllsedimente. Am Eingang dieses Höhlenteils fanden sich inmitten dieser Verfüllung drei Faunenakkumulationen übereinander, die vom Liegenden zum Hangenden mit F, E und D bezeichnet wurden. Der hier zunächst behandelte Komplex F lag etwa 1,3 m über der „Nullinie“. Nach den Angaben von Herrn Forstmeyer fanden sich in F der Schädel und zahlreiche Skeletteile eines Rothirsches. Auffallenderweise lag der Schädel so, daß die Geweihe im Sediment nach oben standen, woraus zu schließen ist, daß das Tier hier am Grunde des offenen, zu dieser Zeit etwa $5 \mathrm{~m}$ tiefen Schachtes verendete. Das Höhlendach war also zu dieser Zeit im Bereich der „Schwarzen Wand" schon weitgehend eingestürzt. Die Kleinsäuger aus diesem Komplex sind wohl wieder durch Gewölle angereichert worden. Die Zusammensetzung dieser Fauna hat sich gegenüber Komplex $\mathrm{H}$ entscheidend verändert. Trotz der geringen Menge des Materials 
zeigt sich deutlich, daß einerseits alle Arten der glazialen Fauna fehlen und andererseits die wärmeliebenden Arten der postglazialen Faunen bereits dominieren. Neu hinzugekommen ist Apodemus und Pitymys subterraneus. Der Anteil von Cletbrionomys ist gegenüber $\mathrm{H}$ von 3 auf über $50 \%$ angestiegen und zeigt, daß keinerlei glaziale Einflüsse mehr geherrscht haben.

$\mathrm{Da}$ im Komplex $\mathrm{F}$ bereits eine rein holozän geprägte Fauna vorliegt, ist der Abschluß des Faunenaustausches am Ende des letzten Glazials zwischen den Komplexen $\mathrm{H}$ und $\mathrm{F}$ anzusetzen. Nach der C14-Datierung der überlagernden Komplexe $\mathrm{E}$ und $\mathrm{D}$, die beide in die erste (ältere) Hälfte des 10. Jahrtausends BP fallen, ergibt sich, daß der Komplex F eine besonders frühe Fauna nach diesem Austausch darstellt. Faunistisch könnte man diese Einstufung nicht so scharf eingrenzen. Pitymys subterraneus und die Schnecke Discus ruderatus lassen das Alter nur auf Alt- und Mittelholozän eingrenzen.

\section{Tabelle 2}

Euerwanger Bühl. Faunenzusammensetzung im Komplex F.

\section{Gastropoda}

Discus ruderatus

Clausilia dubia

Bradybaena fruticum

1 Exemplar

1

Anura indet.

Amphibia indet.

Reptilia

Natrix sp.

Anguis fragilis

Aves indet.

Mammalia

Sorex araneus

Sorex minutus

Talpa europaea

Chiroptera indet.

Cletbrionomys glareolus

Arvicola terrestris

Pitymys subterraneus

Microtus arvalis-agrestis

Apodemus flavicollis

Apodemus sylvaticus

Mustela nivalis

Cervus elaphus

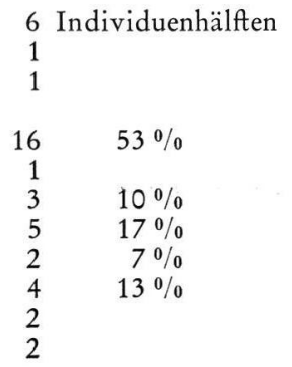

$100 \%(\mathrm{n}=30)$

Da die artliche Zusammensetzung von Komplex $\mathrm{F}$ fast der von $\mathrm{C}$ entspricht, dieser aber wesentlich individuenreicher ist, sollen die faunistischen Besonderheiten gemeinsam besprochen werden. Die Schneckenfauna ist im Komplex F sehr spärlich und enthält nur je ein Exemplar von Bradybaena fruticum, Clausilia dubia und der für das Altholozän typischen Art Discus ruderatus.

\section{Komplexe E und D}

Die Komplexe $\mathrm{E}$ und $\mathrm{D}$ wurden $1-2 \mathrm{~m}$ über dem Komplex $\mathrm{F}$ und damit 2,5-3,5 $\mathrm{m}$ über der „Nullinie“ aufgesammelt. Etwa $3 \mathrm{~m}$ weiter nordöstlich wurde Komplex C in den gleichen, Holzkohle führenden Lagen aufgesammelt. Während die Großfauna mehr oder weniger getrennt gehalten wurde, ist die Kleinfauna der drei Komplexe möglicherweise vermischt worden. Zum vorwiegenden Teil stammt die Kleinfauna jedoch sicher aus C. 
Der dadurch entstandene Informationsverlust ist zwar bedauerlich, aber da alle drei Komplexe nach den $\mathrm{C}^{14}$-Datierungen innerhalb des 10 . Jahrtausends BP liegen, kann man diesen Sammelkomplex durchaus noch zur Klärung der Faunenentwicklung des Altholozäns heranziehen.

An Großsäugern lieferten die Komplexe E und D

$\begin{array}{ll}\text { Canis lupus } & \text { Wolf } \\ \text { Meles meles } & \text { Dachs } \\ \text { Cervus elaphus } & \text { Rothirsch } \\ \text { Capreolus capreolus } & \text { Reh } \\ \text { Bos primigenius } & \mathrm{Ur}\end{array}$

Der Wolf (Canis lupus) im Komplex D ist durch den linken Unterkiefer eines Jungtieres kurz vor dem Zahnwechsel belegt. Der Eckzahn sowie die drei Molaren sind im Kiefer bereits ausgebildet aber noch nicht herausgeschoben. Die Länge des herauspräparierten unteren ersten Molaren beträgt 27,1 mm und liegt damit im Variationsbereich des Wolfes.

Der Dachs (Meles meles) ist durch einen Eckzahn und der Hirsch (Cervus elaphus) durch einige Molaren sowie das Fragment einer Abwurfstange belegt. Die Aufnahme des Rehs (Capreolus capreolus) in die Faunenliste beruht auf einer mündlichen Mitteilung von Prof. Heller, Erlangen, an den Ausgräber. Uns haben für diese Art keine Belege vorgelegen. Vom Ur (Bos primigenius) sind drei Schneidezähne und ein Prämolar vorhanden. In die Faunenliste wurde das Rentier (Rangifer tarandus) $\mathrm{n} \mathrm{icht} \mathrm{aufgenommen,} \mathrm{da} \mathrm{die}$ beiden Molaren, die angeblich aus Komplex D stammen, sich in der Erhaltung von dem übrigen Material erheblich unterscheiden und dem der pleistozänen Komplexe entsprechen. $\mathrm{Da}$ in keiner der neuen, mit exakten Methoden gegrabenen Stationen Süddeutschlands ein Nebeneinander von Reh und Rentier im Altholozän beobachtet werden konnte, scheint hier eine Verwechslung wahrscheinlicher als ein Wiederauftreten von Rangifer zusammen mit Capreolus. Bei einem Teil der Großsäuger kann es sich um die Jagdbeute des Menschen handeln. Diesen Zusammenhang machen die Funde von Artefakten sowie die Konzentrationen von Holzkohle wahrscheinlich. Der Kiefer des jungen Wolfes lag nach Angaben des Finders nahe einer solchen „Feuerstelle“.

$$
\text { Komplex C }
$$

Die Kleinfauna stammt wiederum aus Gewöllen. Wie oben schon erwähnt wurde, ist nicht auszuschließen, daß einige der hier aufgeführten Funde aus den Fundkomplexen D und E stammen.

Es handelt sich hier wie im Komplex F um eine reine Holozän-Fauna, die sich grundsätzlich von derjenigen des Komplexes $\mathrm{H}$ unterscheidet. Wie in $\mathrm{F}$ ist Cletbrionomys am häufigsten. Sie wird von anderen holozänen Leitarten wie Apodemus sylvaticus, A. flavicollis und Pitymys subterraneus begleitet. Innerhalb der Microtus arvalis-agrestis-Gruppe hat sich das Häufigkeitsverhältnis gegenüber $\mathrm{H}$ auffallend verschoben. Microtus agrestis scheint hier etwa gleich häufig wie $M$. arvalis vertreten zu sein.

Besonderes Augenmerk verdienen die Arvicola-Reste. Metrisch sind die aus Komplex C - ebenso wie die aus Komplex A2 - deutlich kleiner als aus dem kaltzeitlichen Komplex H. Das bezieht sich nicht nur auf die Abmessungen des $M_{1}$ (Abb. 2), sondern generell auch auf die Langknochen, die allerdings wegen der unterschiedlichen Wachstumsstadien schlechter zu vermessen sind. Es ist nicht das erste Mal, daß ein starker Größenunterschied zwischen Arvicola-Resten aus dem letzten Glazial und dem Postglazial beobachtet wurde. Während Jánossy (1959) hier eine in n e r a r t li c h e Größenverschiebung sieht, nimmt Storch $(1973,1974)$ z we i unterschiedliche Arten an, wozu er vor allem mor- 


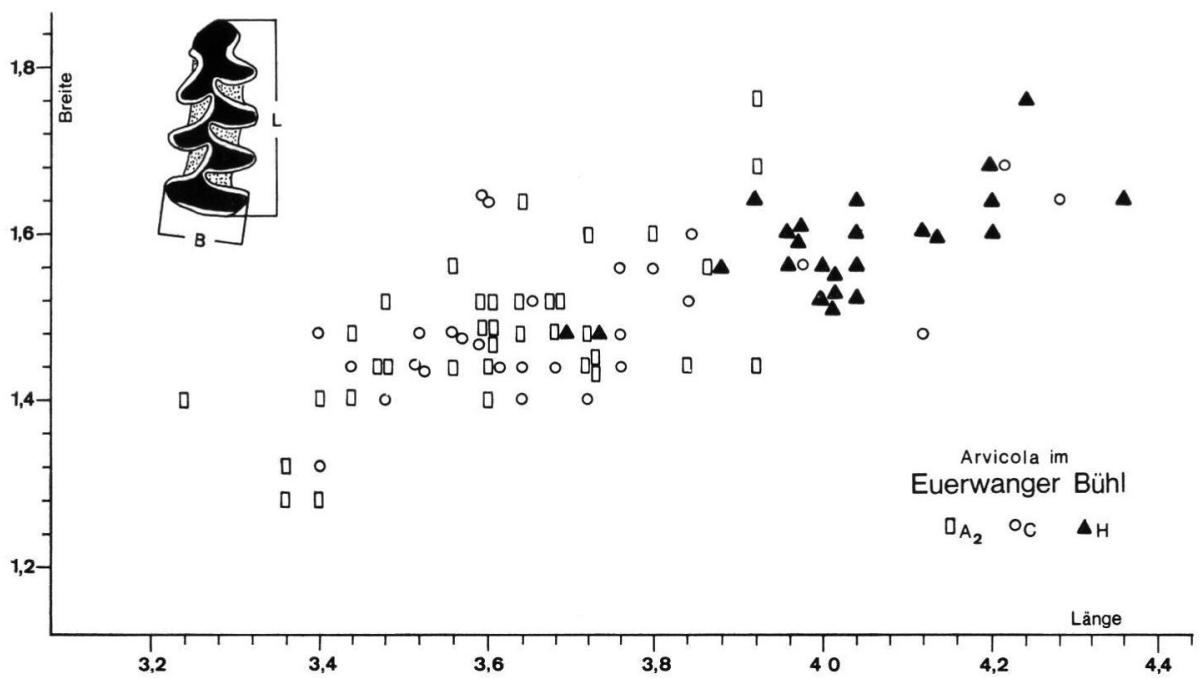

Abb. 2. Euerwanger Bühl. Arvicola terrestris, Längen/Breiten Diagramm der $\mathrm{M}_{1}$ aus dem jungpleistozänen Komplex H und den altholozänen Komplexen C und A 2.

phologische Merkmale heranzieht. Die kaltzeitliche Großform bezeichnet er A.antiquus Pomel 1853, die postglaziale Kleinform stellt er zur rezenten A terrestris L. 1758.

Tabelle 3

Euerwanger Bühl. Faunenzusammensetzung im Komplex C (mit D und E ?)

\begin{tabular}{|c|c|c|}
\hline \multicolumn{3}{|l|}{ Gastropoda } \\
\hline Bradybaena fruticum & \multicolumn{2}{|c|}{1 Exemplar } \\
\hline \multicolumn{3}{|l|}{ Reptilia } \\
\hline \multicolumn{3}{|l|}{$\begin{array}{l}\text { Natrix natrix } \\
\text { Anguis fragilis }\end{array}$} \\
\hline \multicolumn{3}{|l|}{ Aves indet. } \\
\hline \multicolumn{3}{|l|}{ Mammalia } \\
\hline Sorex araneus & 7 & Individuenhälften \\
\hline Talpa europaea & 9 & \\
\hline Chiroptera indet. & & \\
\hline Cletbrionomys glareolus & 56 & $46 \%$ \\
\hline Arvicola terrestris & 36 & \\
\hline Pitymys subterraneus & 7 & $6 \%$ \\
\hline Microtus arvalis-agrestis & 51 & $42 \%$ \\
\hline Apodemus flavicollis & 3 & $2 \%$ \\
\hline Apodemus sylvaticus & 5 & $4 \%$ \\
\hline Muscardinus avellanarius & 3 & \\
\hline Mustela nivalis & 2 & \\
\hline & & $100 \%(\mathrm{n}=121)$ \\
\hline
\end{tabular}

Von den Unterscheidungsmerkmalen werden hier die des Unterkiefers hervorgehoben, da sie am vorliegenden Material überprüft werden können. Die Form des Vorderlobus variiert bei Arvicola erheblich, SтоRсн betont, daß linguale und labiale Kanten am Vorderlobus für „antiquus" kennzeichnend sind, während sie in postglazialem Material nur 
sehr selten auftreten. Bei dem vorliegenden Material konnten Kanten sowohl im Komplex $\mathrm{H}$ als auch in gleicher Ausprägung und ähnlicher Häufigkeit in den postglazialen Komplexen C und A2 beobachtet werden. Die Kantenausbildung, die unabhängig von der Zahnlänge vorhanden ist, läßt keine Unterscheidung der Populationen zu und ist damit $\mathrm{k}$ e in Beweis für eine genetische Isolierung.

Ein weiteres Unterscheidungsmerkmal wird für den Unterkiefer angegeben. Die Form des Processus articularis ist bei „antiquus" gestreckt, während sie bei "terrestris" eingeknickt ist (vgl. SтоRсн 1974, Abb.4). Dies Merkmal erweist sich deutlich als größenabhängig. So zeigen Unterkiefer mit langen $\mathrm{M}_{1}$ die gestreckte Form, Unterkiefer mit kurzem $\mathrm{M}_{1}$ eine stark geknickte Form. Da die durchschnittliche Kiefergröße in $\mathrm{H}$ höher liegt als in $\mathrm{C}$ und A2, ist die gestreckte Form dort zwar häufiger, da sich aber in C und A2 die gleiche Tendenz abzeichnet, ist dieser Unterschied nicht grundsätzlich, sondern nur graduell. Dazu paßt die Beobachtung von STоRсн $(1974,351)$ bestens, daß bei rezentem süddeutschem Material, das im Durchschnitt von größeren Individuen stammt als das aus dem Altholozän, wieder eine Annäherung an die Form von „antiquus" zu beobachten ist.

Durch die Feststellung, daß die Kantenbildung sich in den hier behandelten Komplexen $\mathrm{H}$ einerseits und $\mathrm{C}$ und $\mathrm{A} 2$ andererseits nicht grundsätzlich unterscheidet, und daß die Form des Processus articularis eine Funktion der Größe darstellt, ist es unmöglich, die kaltzeitliche Großform und die postglaziale Kleinform als genetisch unabhängige Arten einander gegenüberzustellen. Ein Nebeneinander beider Arten in allen Komplexen würde nach der Größenverteilung der $\mathrm{M}_{1}$ (Abb. 2) erfordern, daß beide Arten an der PleistozänHolozän-Grenze eine gleichsinnige Größenverschiebung erfahren, was aber höchst unwahrscheinlich sein dürfte.

Wenn sich hier unseres Erachtens nach auch keine genetische Isolierung feststellen läßt, weswegen eine Unterscheidung im Artniveau zu unterbleiben hat, so ist damit keineswegs gesagt, daß die Umbildung von der glazialen Großform zur postglazialen Kleinform innerhalb einer Population am Ort stattgefunden haben muß. Es erscheint durchaus möglich, daß die Faunenverschiebungen am Ende des Glazials das Einwandern neuer Rassen bewirkten, nur war die genetische Isolierung nicht so vollständig, daß die Merkmale der Großform, wie die Kantenbildung am Vorderlobus des $\mathbf{M}_{1}$, nicht weitergegeben werden konnten. Es scheint, daß die ökologischen Verhältnisse, die während des letzten Glazials in Süddeutschland große Varianten von A.terrestris begünstigten, im Altholozän den kleinen den Vorteil gaben.

\section{Komplex B}

An der Nordwand des als „Schwarze Wand“ bezeichneten ehemaligen Höhlenarmes ist eine senkrechte Kluft durch Auslaugung zu einer Spalte erweitert. In ihrem oberen Teil, etwa 3,5 m über der "Nullinie“, bildet sie sogar eine kleine Höhlung aus. Hier fanden sich die Faunenreste des Komplexes B. In der Tiefe der Spalte wurden weitere Faunenreste aufgesammelt. Da sie sich jedoch als stratigraphisch uneinheitlich herausstellten, können sie hier übergegangen werden.

Das C14-Datum dieser kleinen Fauna beträgt $9190 \pm 90$ BP. Damit gehört diese Fauna in das Präboreal. Der altholozäne Charakter zeichnet sich in der Kleinsäugerfauna, die wohl ebenfalls Gewöllen entstammt, sehr deutlich ab. Cletbrionomys und Apodemus sind vorhanden. Rein glaziale Faunenelemente fehlen. Bei dem einzigen Relikt aus der pleistozänen Fauna, dem Hamster (Cricetus cricetus), handelt es sich um eine sehr anpassungsfähige Art, die in Süddeutschland und in der Schweiz stellenweise auch noch als Mitglied altholozäner Faunen angetroffen wird. 
Tabelle 4

Euerwanger Bühl. Faunenzusammensetzung im Komplex B

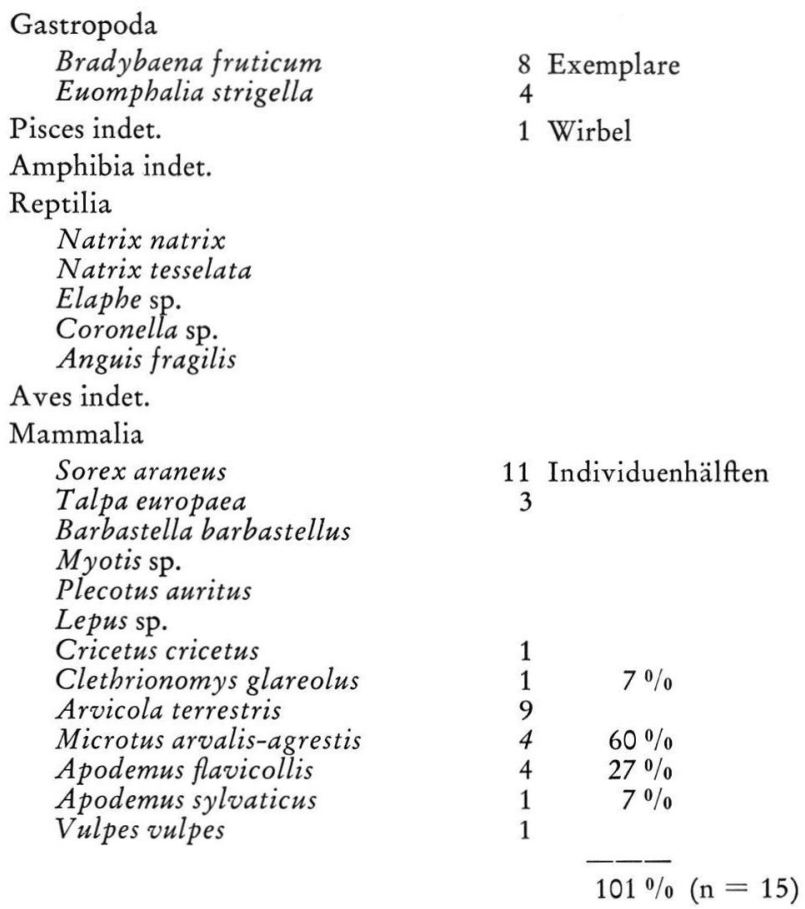

Die Fledermausreste müssen altersmäßig nicht unbedingt mit dem C14-Datum übereinstimmen. Dem Dolomitsand, in dem die Tierreste oberflächlich eingebettet waren, fehlt eine Abdeckung durch jüngere Sedimente, so daß die Fledermausreste auch aus späterer Zeit stammen können.

\section{Komplex A}

Der Fundkomplex A liegt im hinteren Teil der „Schwarzen Wand“. Er befindet sich etwa 4 bis 4,5 m über der "Nullinie" und wurde in einen unteren Abschnitt A2 und einen oberen Abschnitt A1 unterteilt. Für A2 liegt eine C14-Datierung mit $8790 \pm 110$ BP vor. Auf Grund der Gliederung des Holozäns von TAUBer (1970) gehört A2 damit bereits in das Boreal. Die zeitliche Einstufung von A1, das A2 ohne erkennbare Sedimentationsunterbrechung überlagert, wird weiter unten diskutiert.

Der Artenbestand an Säugetieren ist in A2 und A1 rein holozän geprägt und enthält keinerlei kaltzeitliche Reliktarten. Im Unterschied $\mathrm{zu}$ den Komplexen $\mathrm{C}$ und $\mathrm{F}$ ist hier mit Crocidura leucodon eine weitere holozäne Leitart vertreten. Grundsätzlich sind jedoch im Artenbestand bei einem Vergleich mit den älteren holozänen Komplexen keine wesentlichen Veränderungen zu erkennen. Feststellen läßt sich nur eine gewisse Verschiebung in der quantitativen Verteilung der mittelgroßen Nager: während Clethrionomys in $\mathrm{F}$ und $\mathrm{C}$ noch einen Anteil von 50\% und mehr erreicht, geht ihr Anteil in A2, und noch stärker in A1, bedeutend zurück. Gleichzeitig nimmt der Anteil von Apodemus flavicollis zu, während derjenige von Apodemus sylvaticus stets gering bleibt. 
Den relativ geringfügigen Veränderungen innerhalb der Säugetierfaunen des Komplexes A steht ein starker Wandel in den Gastropodenfaunen gegenüber. In A2 liegt eine klassische "Discus ruderatus-Fauna" vor, in der Discus rotundatus nur vereinzelt eingestreut ist. In A1 dagegen sind beide Arten gleich häufig und es hat den Anschein, daß hier das plötzliche Zurückgehen der sibirisch-asiatischen Waldart Discus ruderatus belegt ist, das LožEK (1964) für die ČSSR zwischen dem älteren und jüngeren Atlantikum ansetzt und für das ANT (1965) zunehmende Wärme und Feuchtigkeit im Atlantikum und möglicherweise auch die Konkurrenz neu einwandernder süd- und westeuropäischer Formen verantwortlich macht.

Tabelle 5

Euerwanger Bühl. Vertebratenfauna in den Komplexen A 2 und A 1

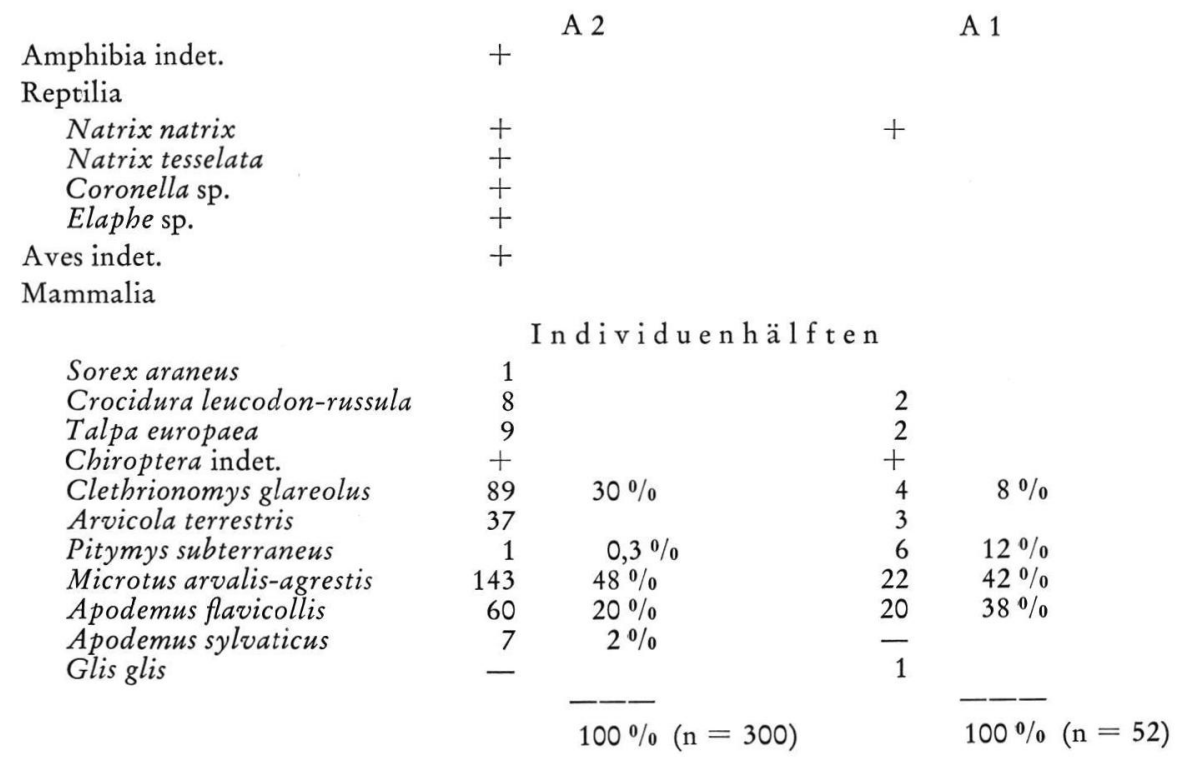

Es erscheint sehr fraglich, ob diese auf die ČSSR bezogene Zeitmarke auch für Süddeutschland gültig ist. Es wurde oben schon erwähnt, daß zwischen A2, das in das frühe Boreal zu stellen ist, und dem überlagernden Abschnitt A1 keine Sedimentationslücke zu erkennen war. Eine solche Sedimentationspause, die im vorliegenden Fall etwa eineinhalb Jahrtausende umfassen müßte, wäre schon aus topographischen Gründen unwahrscheinlich, da sich eine offene Hohlform, wie sie die "Schwarze Wand“ zu jener Zeit bildete, kontinuierlich und schnell verfüllt. Damit ist ein größerer Altersunterschied zwischen A2 und $\mathrm{A} 1$ auszuschließen.

Zur Zeit ist noch nicht abzusehen, ob hier lokale Veränderungen in der Gastropodenfauna stratigraphische Zonierungen größeren Ausmaßes vortäuschen oder ob man in gewissen süddeutschen Gebieten mit einem früheren Verschwinden von Discus ruderatus rechnen muß, als es LožEK für die ČSSR festgestellt hat.

In der Schneckenfauna des Abschnittes A2 sind zwei Arten ausgesprochen dominant: Discus ruderatus und die Schließmundschnecke Clausilia dubia. Die letztere Art dürfte in großer Zahl die Felsen der „Schwarzen Wand“ besiedelt haben.

Im Artenbestand fällt eine größere Zahl xero- und thermophiler und meist offenes Gelände bevorzugender Formen auf. Hierher gehören Cochlicopa lubricella, Abida fru- 
Tabelle 6

Euerwanger Bühl. Gastropodenfauna der Komplexe A und R

\begin{tabular}{|c|c|c|c|c|c|c|c|c|c|}
\hline Euerwanger Bühl & $\begin{array}{l}\mathrm{K} \text { o } \mathrm{m} \\
\mathrm{A} 2\end{array}$ & ${ }^{x}$ A 1 & R VII & R VI & $\mathrm{R} \mathrm{V}$ & $\underset{\text { R IV }}{\operatorname{Komp}}$ & R III & $\mathrm{R}$ II & R I \\
\hline \multicolumn{10}{|l|}{ 1. Wald } \\
\hline ! Acanthinula aculeata & 1 & - & - & 1 & 6 & 13 & 11 & - & - \\
\hline ! Aegopinella pura & - & 2 & - & 1 & - & 17 & 16 & 2 & - \\
\hline ! Cochlodina laminata & 2 & - & - & - & - & - & - & - & - \\
\hline - Discus ruderatus & ca. 200 & 10 & 102 & 215 & 226 & 627 & 520 & 87 & 88 \\
\hline ! Ena obscura & 5 & 2 & - & - & 3 & 22 & 18 & - & - \\
\hline ! Helicodonta obvoluta & 4 & 3 & - & - & - & - & - & - & - \\
\hline ! Isognomostoma isognom. & - & 3 & - & - & - & - & - & - & - \\
\hline ! Monachoides incarnata & 1 & 3 & - & - & - & 1 & - & - & - \\
\hline \multirow[t]{2}{*}{ - Vertigo pusilla } & - & - & - & 1 & 7 & 6 & 5 & - & - \\
\hline & $\begin{array}{l}\text { ca. } 213 \\
(37,0 \%)\end{array}$ & $\begin{array}{c}23 \\
(21,1 \%)\end{array}$ & $\begin{array}{c}102 \\
(25,7 \%)\end{array}$ & $\begin{array}{c}218 \\
(42,4 \%)\end{array}$ & $\begin{array}{c}242 \\
(39,1 \%)\end{array}$ & $\begin{array}{c}686 \\
(41,5 \%)\end{array}$ & $\begin{array}{c}570 \\
(34,1 \%)\end{array}$ & $\begin{array}{c}89 \\
(56,3 \%)\end{array}$ & $\begin{array}{c}88 \\
(72,7 \%)\end{array}$ \\
\hline
\end{tabular}

2. Wald, Gebüsch, Waldsteppe und offene, mehr oder weniger feuchte Standorte

\begin{tabular}{|c|c|c|c|c|c|c|c|c|c|}
\hline ! Aegopinella nitens/minor & 20 & 8 & - & - & - & 54 & 23 & 15 & 12 \\
\hline Bradybaena fruticum & 13 & 6 & 2 & 5 & 6 & 4 & 4 & - & 1 \\
\hline ! Cepaea hortensis & - & - & - & - & - & 1 & 1 & - & - \\
\hline Discus rotundatus & 4 & 10 & - & - & - & 2 & 2 & - & 1 \\
\hline Helicigona arbustorum & - & - & 1 & 1 & 2 & 1 & 2 & 1 & 1 \\
\hline Limax cinereoniger & 8 & 12 & - & 3 & 20 & 13 & 21 & 7 & - \\
\hline \multirow[t]{2}{*}{ Vitrea crystallina } & - & - & - & - & - & - & 10 & - & - \\
\hline & $\begin{array}{c}45 \\
(7,8 \%)\end{array}$ & $\begin{array}{c}36 \\
(33,0 \%)\end{array}$ & $\begin{array}{c}3 \\
\left(0,7^{0} \%\right)\end{array}$ & $\begin{array}{c}9 \\
\left(1,7^{0} / 0\right)\end{array}$ & $\begin{array}{c}28 \\
\left(4,5^{\circ} \%\right)\end{array}$ & $\begin{array}{c}75 \\
(4,5 \%)\end{array}$ & $\begin{array}{c}63 \\
(3,8 \%)\end{array}$ & $\begin{array}{c}23 \\
(14,6 \%)\end{array}$ & $\begin{array}{c}15 \\
(12,4 \%)\end{array}$ \\
\hline
\end{tabular}

3. Steppe und Felssteppe

- Abida frumentum

* Cecilioides acicula

Chondrula tridens

Helicella obvia

* Zebrina detrita

\begin{tabular}{lccccccc}
2 & 6 & 4 & 5 & 14 & 22 & 1 & - \\
- & - & - & - & 1 & - & - & - \\
- & 2 & - & - & - & - & - & - \\
- & - & - & - & 2 & - & - & - \\
\hline 2 & 8 & 9 & 6 & 20 & 25 & 1 & -
\end{tabular}


4. Offenes Gelände

- Enomphalia strigella

- Pupilla muscorum

Truncatellina cylindrica

Vallonia costata

- Vallonia excentrica

- Vallonia pulchella

- Vertigo pygmaea

\begin{tabular}{|c|c|c|c|c|c|c|c|c|}
\hline 6 & 10 & - & 2 & 9 & 22 & 24 & 7 & 1 \\
\hline- & - & 1 & - & - & - & - & - & - \\
\hline- & - & - & 5 & 2 & 1 & 1 & - & - \\
\hline 24 & 1 & 101 & 80 & 94 & 225 & 227 & 1 & 4 \\
\hline- & - & - & 1 & - & 1 & 2 & - & - \\
\hline- & - & 3 & - & 2 & 6 & 5 & - & - \\
\hline- & - & 3 & - & 2 & 2 & - & - & - \\
\hline $\begin{array}{l}0 \\
2 \%)\end{array}$ & $\begin{array}{c}11 \\
(10,1 \%)\end{array}$ & $\begin{array}{c}108 \\
(27,2 \%)\end{array}$ & $\begin{array}{c}88 \\
(17,1 \%)\end{array}$ & $\begin{array}{c}109 \\
(17,6 \%)\end{array}$ & $\begin{array}{c}257 \\
(15,5 \%)\end{array}$ & $\begin{array}{c}259 \\
(15,5 \%)\end{array}$ & $\begin{array}{c}8 \\
(5,1 \%)\end{array}$ & $\begin{array}{c}5 \\
(4,1 \%)\end{array}$ \\
\hline
\end{tabular}

5. Offenes und bewaldetes Gelände (trocken)

- Cocblicopa lubricella

$$
(3,7 \%)
$$

3
$(0,7 \%$

10
$(1,9 \%$

16
$(2,6 \%)$

45
$(2,7 \%)$

36
$(2,0 \%)$

$(0,6 \%)$

$\left(0,8^{1} \%\right)$

6. Offenes und bewaldetes Gelände (mäßig feucht)

- Abida secale

Clausilia dubia

- Euconulus fulvus

! Helicigona lapicida

Limacidae (kleine Arten)

- Nesovitrea hammonis

Punctum pygmaeum

- Trichia hispida

Vitrea contracta

- Vitrina pellucida

ca. $200 \quad \overline{17}$

$\overline{17}$

$\overline{17}$

$\frac{-}{115}$
$\frac{-}{40}$
$\frac{7}{4}$
$\frac{3}{-}$
169
$42,5 \%)$

$\begin{array}{cc}1 & - \\ 93 & 119 \\ 3 & 2 \\ \overline{65} & -\overline{44} \\ 3 & 17 \\ 4 & 5 \\ 2 & 4 \\ 1 & 5 \\ - & 1 \\ 172 & 197 \\ (33,4 \%) & (31,9 \%)\end{array}$

$\begin{array}{rrrr}3 & - & - & - \\ 264 & 224 & 33 & 3 \\ 9 & 5 & - & - \\ -5 & \overline{-} & - & - \\ 75 & 112 & - & 1 \\ 63 & 44 & - & - \\ 5 & 5 & - & 1 \\ 3 & 2 & - & - \\ 101 & 252 & 36 & 12 \\ 6 & 3 & (22,8 \%) & (10,0 \%) \\ 529 & 647 & & \end{array}$

7. Offenes und bewaldetes Gelände (feucht)

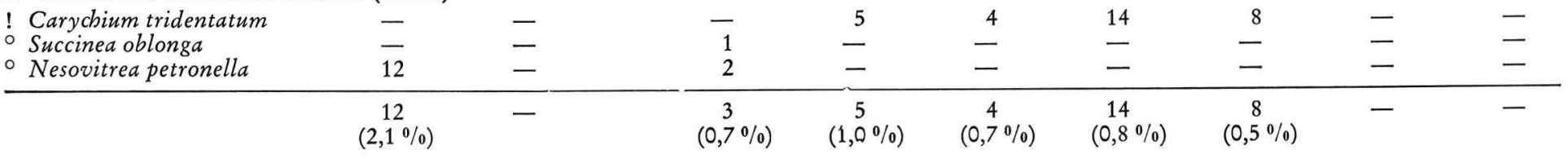

8. Sehr feuchte bis nasse Standorte

- Carychium minimum

\begin{tabular}{|c|c|c|c|c|c|c|c|c|c|}
\hline - Carychium minimum & 一 & - & $\begin{array}{c}1 \\
(0,3 \%)\end{array}$ & $\left.\begin{array}{c}4 \\
(0,8\end{array} \%\right)$ & $\begin{array}{c}16 \\
(2,6 \%)\end{array}$ & $\begin{array}{c}28 \\
(1,7 \%)\end{array}$ & $\begin{array}{c}68 \\
(4,0 \%)\end{array}$ & - & - \\
\hline $\begin{array}{l}\text { Individuenzahl }(=100 \%) \\
\text { Artenzahl }\end{array}$ & $\begin{array}{r}575 \\
27\end{array}$ & $\begin{array}{r}109 \\
18\end{array}$ & $\begin{array}{r}397 \\
18\end{array}$ & $\begin{array}{r}515 \\
24\end{array}$ & $\begin{array}{r}618 \\
25\end{array}$ & $\begin{array}{r}1654 \\
34\end{array}$ & $\begin{array}{r}1676 \\
31\end{array}$ & $\begin{array}{r}158 \\
12\end{array}$ & $\begin{array}{r}121 \\
13\end{array}$ \\
\hline
\end{tabular}


mentum, Chondrula tridens, Vitrea contracta und Euomphalia strigella. Daneben finden sich Waldschnecken, die durch eine Reihe wenig anspruchsvoller Arten wie Acantbinula aculeata, Discus rotundatus, Cochlodina laminata, Ena obscura, Limax cinereoniger, Aegopinella sp. und Monachoides incarnata vertreten sind. Eine ausgesprochen wärmeliebende, südliche Waldart ist Helicodonta obvoluta.

Das Artenspektrum weist auf einen relativ trockenen, mit freien Flächen durchsetzten Waldbiotop hin, in dem sowohl die offenes Gelände bevorzugenden Arten als auch die Waldformen ausreichende Lebensbedingungen vorfanden.

Problematisch ist das Vorkommen einiger Arten, die offenbar erst im späten Holozän feste Bestandteile der mitteleuropäischen Malakofauna geworden sind und die in altholozänen Sedimenten bisher noch nicht mit Sicherheit nachgewiesen werden konnten. In erster Linie gilt dies für die mediterrane Art Zebrina detrita, aber auch für die im weitesten Sinne pontische Helicella obvia. Nach LožEK (1964) treten diese beiden xerothermen Schnecken in Mitteleuropa frühestens im Jungholozän auf, so daß ihr Vorkommen in den altholozänen Ablagerungen des Euerwanger Bühls auf nachträgliche Verlagerung von Gehäusen in tiefere Schichten zurückgeführt werden müßte. Wenn diese Möglichkeit auch durchaus besteht, so finden wir doch andererseits in Höhlenablagerungen im Gebiet der Schwäbischen Alb, die borealen Alters sind (Falkenstein bei Thiergarten, HaEssLeIN 1952; Burghöhle bei Dietfurt, RäHLE unveröff.), mit Truncatellina callicratis und Jaminia quadridens mediterrane Faunenelemente in der süddeutschen Fauna vor, die viel anspruchsvoller sind als die obengenannten Arten und die heute in Deutschland überhaupt nicht mehr oder nur noch an Wärmeinseln vorkommen. Alle diese Befunde könnten Indizien für einen offenbar sehr warmen und trockenen, wenn auch nur kurzen Klimaabschnitt im Boreal sein. Für diese Ansicht spricht auch die Tatsache, daß Zebrina detrita am Euerwanger Bühl nicht nur in Komplex A, sondern auch innerhalb des gleichaltrigen Komplexes R auftritt (s. S. 173).

Neben der Anwesenheit besonders wärmeliebender Formen ist für A2 das relativ zahlreiche Auftreten der heute nordeuropäisch-sibirischen Art Nesovitrea petronella besonders beachtenswert. Nesovitrea petronella hat sich im Laufe des Holozäns aus den Niederungen Mitteleuropas in die höheren Lagen der Alpen und die Gipfellagen der Mittelgebirge zurückgezogen und ist heute in unserem Gebiet ein ausgesprochen boreoalpines Faunenelement. Hier läßt sich eine Parallele zur Verbreitungsgeschichte von Discus ruderatus aufzeigen, einer Art, die im heutigen Mitteleuropa ebenfalls ein borealpines Faunenelement darstellt, im Altholozän Mitteleuropas jedoch ein geschlossenes Verbreitungsareal besaß, das erst im Laufe des Holozäns durch klimatische Veränderungen disjungiert wurde (ANT 1965).

Die Gastropodenfauna von A1 zeigt gegenüber derjenigen von A2 trotz einer gewissen Ähnlichkeit in den Molluskenspektren (Abb. 3) einige markante Unterschiede:

1. Die sibirische Waldart Discus ruderatus und der subatlantische Discus rotundatus sind gleichhäufig. Eine ausgesprochene Dominanz der ersteren ist nicht festzustellen.

2. Unter den Waldarten befinden sich in A1 mit Isognomostoma isognomostoma eine ausgesprochen hygrophile Form.

3. Das quantitative Verhältnis der beiden ökologischen Gruppierungen - Waldformen einerseits und Formen, die offenes Gelände bevorzugen andererseits - hat sich erheblich verschoben. Der Anteil der Waldarten steigt von nicht ganz $1 / 4$ in A2 auf $1 / 3$ in A1 an, während der Anteil der offenes Gelände bevorzugenden Arten von 1/4 auf ca. 1/6 des Artenbestandes zurückgeht (Abb. 3). Besonders deutlich wird der Unterschied dann, wenn man die wärme- und feuchtigkeitsliebenden Holozän-Arten den mehr oder weniger indifferenten Formen gegenüberstellt wie das in Abb. 5 getan wurde. Auf erstere 
entfallen in A1 42,7\% der ausgezählten Individuen (gegenüber $8 \%$ in A2) und rund $50 \%$ der Arten (gegenüber $33,3 \%$ in A2).

4. Ein letzter Unterschied liegt darin, daß in A1 mediterrane Faunenelemente, wie sie in A2 auftraten und deren Zugehörigkeit zur altholozänen Fauna - wie oben erwähnt zumindest für wahrscheinlich gehalten wird, völlig fehlen (Abb. 5).

Insgesamt läßt sich aus der Zusammensetzung der Molluskenfauna und dem Vergleich mit derjenigen von Abschnitt A2 auf eine deutliche Feuchtigkeitszunahme und damit zusammenhängend auf stärkere Bewaldung zur Zeit der Ablagerung des Abschnittes A1 schließen.

Die Analyse der Holzkohlenreste belegt die folgenden Holzarten: Pinus, Quercus, Acer, Ulmus, Corylus, Pumoideae, Rhamnus, Betula und Salix. Sowohl in A2 wie in A1 dominiert Pinus mit weit mehr als 50\% (vgl. Abb. 7).

\section{Komplex R}

Im hintersten Teil der „Schwarzen Wand“ wurde im Sommer 1973 eine Nachgrabung mit Tübinger Studenten angesetzt. Sie sollte den Hiatus zwischen A2 und A1 graduell erfassen helfen und so einer besseren Untersuchung zugänglich machen. In einem randlich stehengebliebenen Block konnten allerdings nur noch Sedimente erfaßt werden, die nach ihrem Faunencharakter dem Abschnitt A2 aus Komplex A und tieferen Lagen entsprechen. Dieser Sedimentblock wurde, da er keinerlei sedimentologische Unterschiede zeigte, in horizontalen Lagen von $20 \mathrm{~cm}$ Dicke abgetragen, wobei die oberste Abtragung (ausnahmsweise $30 \mathrm{~cm}$ umfassend) als R I, die unterste als R VII bezeichnet wurde.

Tabelle 7

Euerwanger Bühl. Vertebratenfauna im Komplex R (R VII - R I)

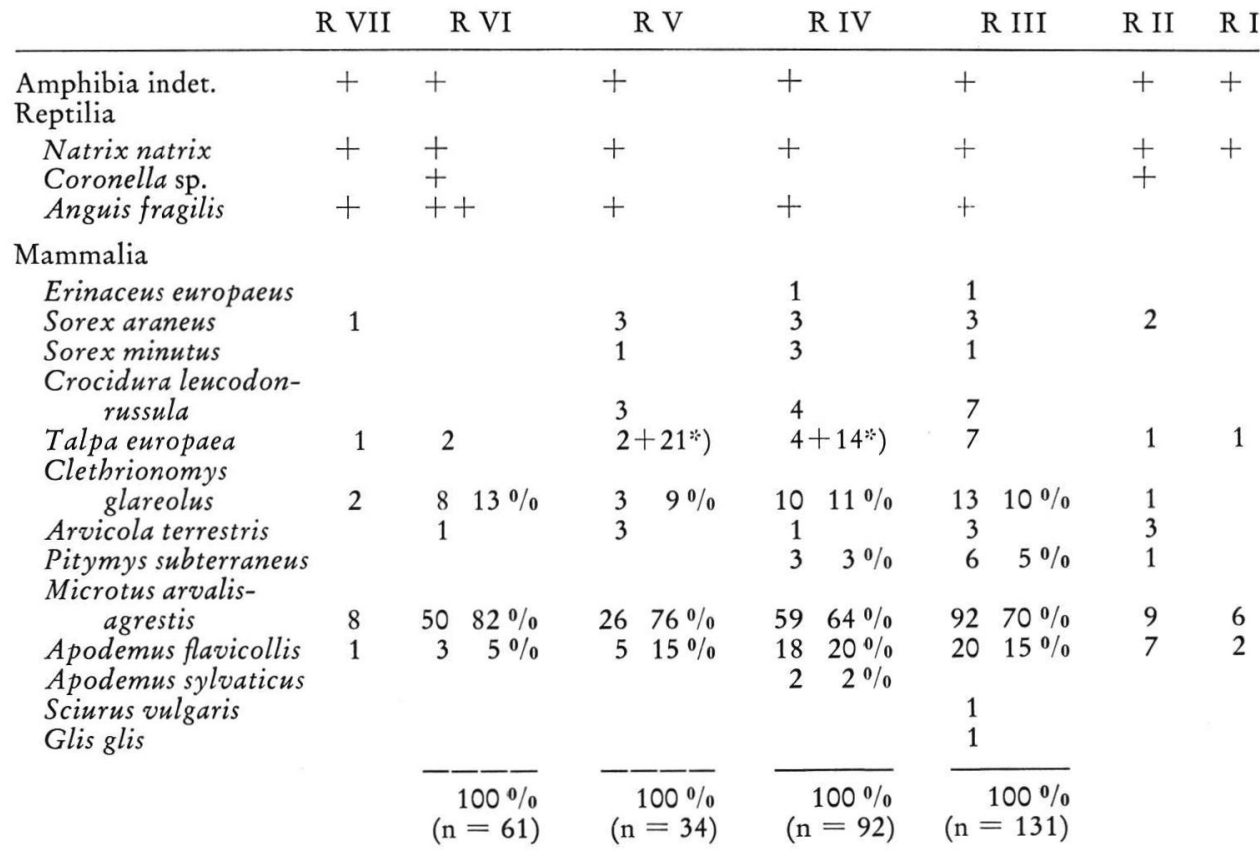

*) Humeri juveniler, Maulwürfe s. S. 172. 


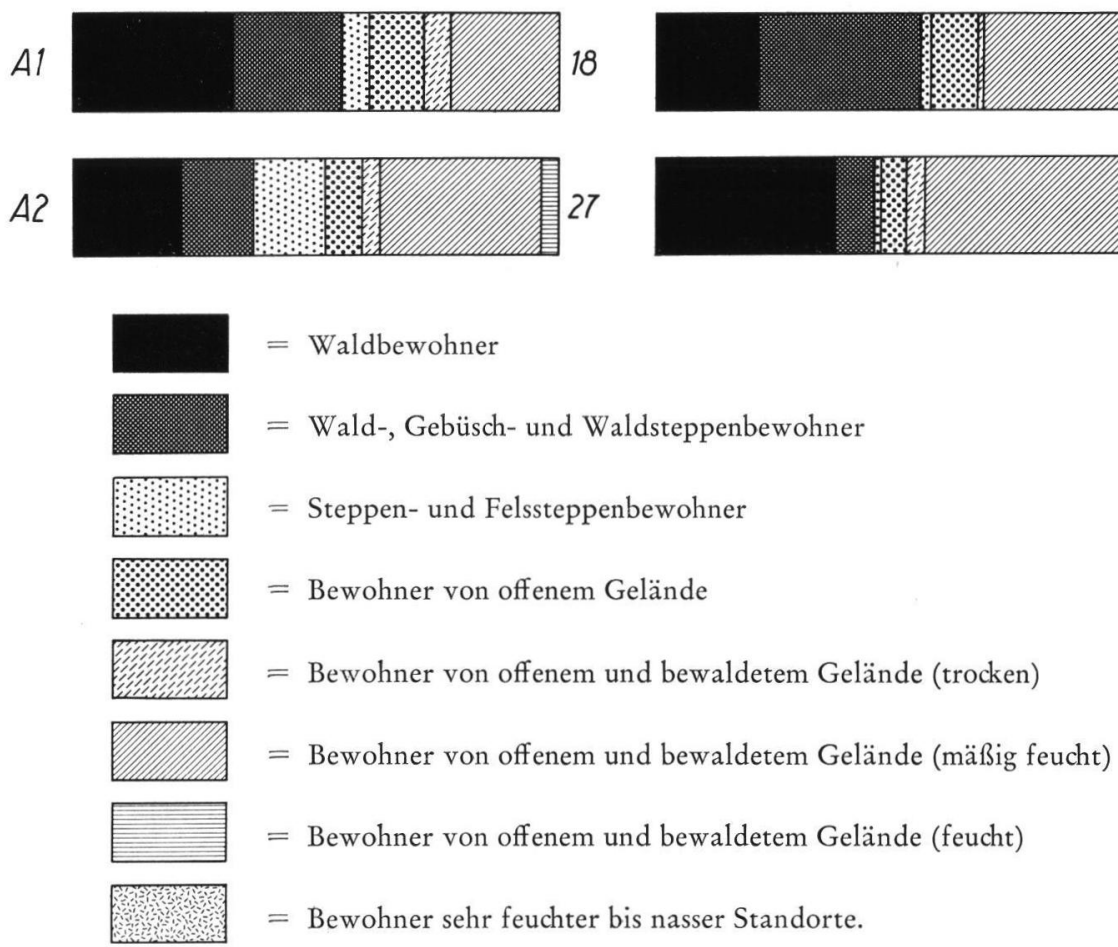

Abb. 3 u. 4. Euerwanger Bühl. Molluskenspektren der Komplexe A und R. Dargestellt ist der Anteil der verschiedenen ökologischen Gruppierungen an der Schneckenfauna, links bezogen auf die Artenzahl, rechts bezogen auf die Anzahl der Individuen.

Al

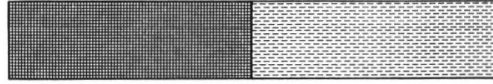

18

$A 2$

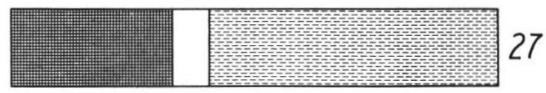

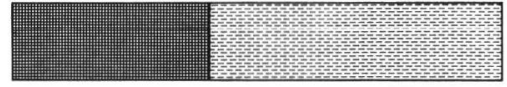

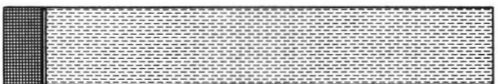

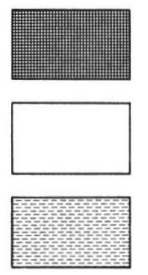

$=$ Arten, die für feucht-warme Klimaabschnitte bezeichnend sind

$=$ Arten, die für trockene und warme Klimate bezeichnend sind (pontische und mediter rane Arten)

$=$ Arten, die klimatischen Gegebenheiten gegenüber mehr oder weniger indifferent sind.

Abb. 5 und 6. Euerwanger Bühl. Molluskenspektren der Komplexe A und R. Dargestellt ist der Anteil verschiedener biost ratigraphischer Gruppen an der Schneckenfauna, links bezogen auf die Artenzahl, rechts bezogen auf die Anzahl der Individuen. 

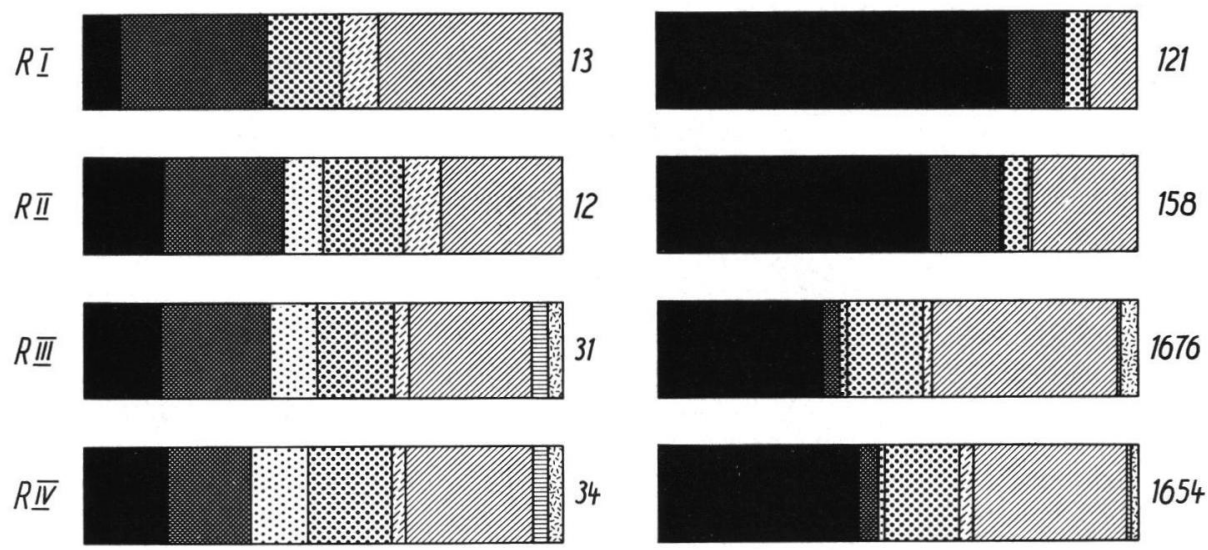

$R \underline{\bar{v}}$
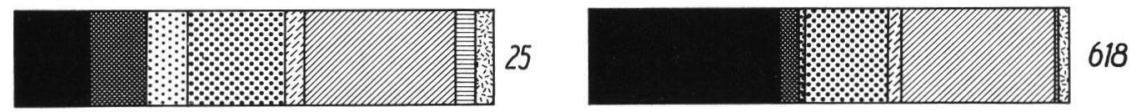

$R \underline{\underline{V}}$
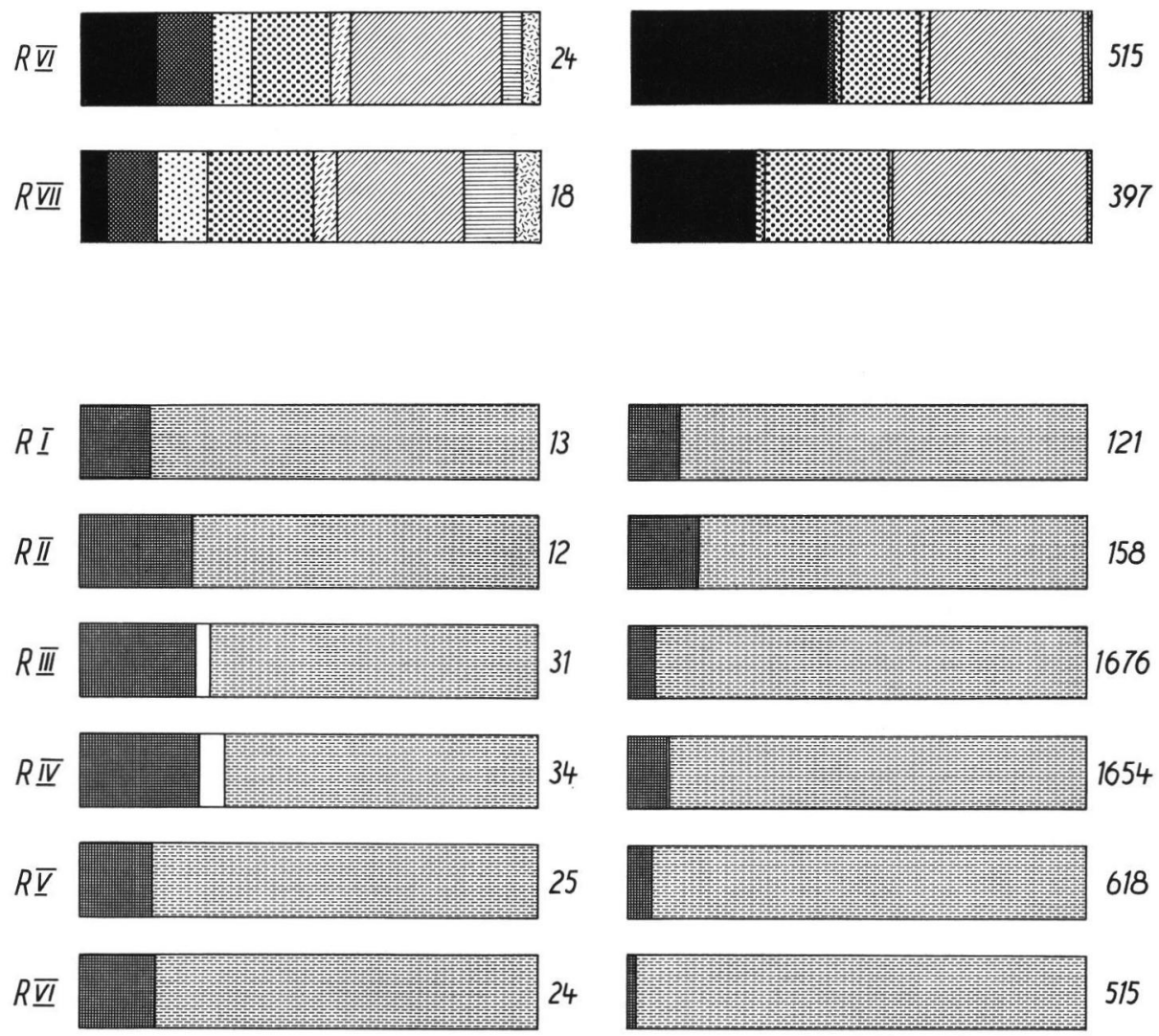

$R \underline{\text { VII }}$
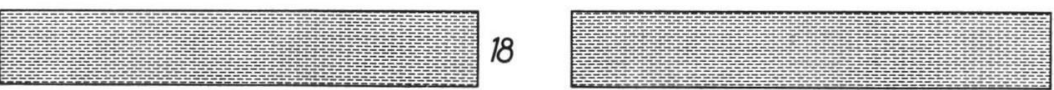
Der Faunenbestand der einzelnen Lagen zeigt, was die Säugetiere betrifft, keine gerichteten Veränderungen. Wiederum handelt es sich um eine rein holozäne Fauna, in der diesmal auch die letzte charakteristische Art, Sciurus vulgaris, für den Euerwanger Bühl belegt werden konnte. Die prozentualen Anteile der mittelgroßen Mäuse lassen sich nicht direkt mit denen der übrigen Komplexe vergleichen, da bei der Nachgrabung das Material weitestgehend geschlämmt wurde, wodurch zahlreiche einzelne Molaren der Microtinen erfaßt wurden, so daß der Anteil von Microtus arvalis-agrestis deutlich höher ist als in den nur oberflächlich aufgesammelten Proben.

Während die Kleinsäugerfaunen der oben besprochenen Komplexe in allen Fällen auf Gewölle zurückzuführen waren, ist in R V und R IV eine Besonderheit zu beobachten. Augenscheinlich wurden in diesen Proben auch Tierbauten, nämlich Maulwurfsgänge, er$\mathrm{faßt}$, in denen Jungtiere umgekommen sind. Auffallend ist die große Zahl jugendlicher Maulwurf-Humeri, die von Tieren stammen dürften, die den Bau noch nicht verlassen hatten und hier ertrunken sind.

Die Schneckenfaunen aller Abtragungen zeichnen sich durch die Dominanz der altholozänen Leitart Discus ruderatus aus. Sie stimmen darin mit der Fauna A2 überein (Tabelle 6). Aus diesem und aus anderen, weiter unten erörterten Gründen ist zu folgern, daß es sich bei $\mathrm{R}$ um einen mit A2 ungefähr gleichaltrigen, also altholozänen Sedimentkomplex handelt.

Ubereinstimmung mit den Schneckenfaunen des Komplexes A besteht im gleichzeitigen Auftreten von zwei ökologischen Gruppierungen, nämlich Waldarten einerseits und Steppenbewohner und Arten des offenen Geländes andererseits, wobei die Wohnplätze der zweiten Gruppe in erster Linie die offenen Kalkfelsen gebildet haben dürften. Die Individuenzahlen der zweiten Gruppe nehmen zu Gunsten der ersten von unten nach oben ab (Abb. 4), was mit der zunehmenden Verfüllung der Höhlenruine in Zusammenhang stehen, vielleicht aber auch die Folge einer allmählichen Feuchtigkeitszunahme sein könnte. Von R VII bis R III bilden feuchtigkeitsliebende Kleinschnecken (in erster Linie Carychien) eine dritte ökologische Gruppe. Als Wohnplätze kommen für sie feuchte Felsnischen und der Nässe speichernde Graben zwischen den Felswänden am Hinterende der „Schwarzen Wand" in Frage.

Die Analyse der Molluskenfaunen ergibt eine Gliederung des Komplexes $\mathrm{R}$ in vier aufeinanderfolgende Zonen ähnlicher ökologischer Gegebenheiten, welche im folgenden näher besprochen werden sollen.

Für R VII ist das Fehlen wärme- und feuchtigkeitsliebender, also anspruchsvoller Holozän-Arten bezeichnend (Abb. 6).

Als Begleiter von Discus ruderatus tritt wie in A2 Nesovitrea petronella auf. Sehr hoch ist der Anteil mehr oder weniger anspruchsvoller Bewohner offenen Geländes wie Vertigo pygmaea, Pupilla muscorum, Abida frumentum, Vallonien und Chondrula tridens, deren Anteile insgesamt 29,4\% der Individuen und 33;3\% der Arten ausmachen. Besonders zahlreich ist wieder die Kalkfelsen liebende Form Clausilia dubia vertreten.

Aus der Zusammensetzung der Molluskenfauna läßt sich der Schluß ziehen, daß R VII früher als A2 abgelagert worden ist, in einer Zeit, in der die Bewaldung noch geringer war und die klimatischen Bedingungen insgesamt gesehen noch nicht für die Entwicklung einer anspruchsvolleren Gastropodenfauna ausreichten.

In R VI und R V stimmen die Molluskenspektren so sehr überein (Abb. 4 und 6), daß für beide Abtragungen gleichartige äußere Bedingungen bei ihrer Ablagerung angenommen werden können.

Discus ruderatus dominiert hier stärker als in R VII (41,8 bzw. 36,6\% der Individuen gegenüber 25,5\% in R VII). Die Steppen- und Felssteppenbewohner sowie die Formen 
des offenen Geländes sind etwas schwächer vertreten als in R VII (18,8 bzw. 18,6 \% der Individuen und 25 bzw. $28 \%$ der Arten gegenüber 29,4\% der Individuen und 33,3\% der Arten in R VII), dafür treten hier erstmals einige Arten auf (Carychium tridentatum, Acanthinula aculeata, Ena obscura, Aegopinella pura, Limax cinereoniger), die höhere Ansprüche an die klimatischen Gegebenheiten stellen als sämtliche in R VII vertretenen Formen. Ihr Anteil an der Gesamtfauna (Abb.6) ist allerdings noch gering (1,9 bzw. $5,3 \%$ der Individuen und 16,7 bzw. 16,0\% der Arten). Insgesamt läßt sich aber aus den Arten- und Individuenspektren der Molluskenfaunen R VI und R V auf eine leichte Klimaverbesserung schließen.

In R IV und R III sind sich die Arten- und Individuenspektren (Abb. 4 und 6) wiederum so ähnlich, daß auch hier eine gemeinsame Besprechung angebracht erscheint.

Der Anteil von Discus ruderatus ist unvermindert hoch (Tabelle 6). Besonders stark vertreten sind xero- und thermophile Faunenelemente wie Cochlicopa lubricella, Truncatellina cylindrica, Abida frumentum, Abida secale, Chondrula tridens, Vitrea contracta und Euomphalia strigella. Sie besitzen in R IV einen Anteil von fast $12 \%$, in R III sogar einen solchen von fast $20 \%$ der Individuen. Für relativ trockene und warme Bedingungen zur Zeit der Ablagerung dieser beiden Schichten spricht auch der außerordentliche Individuenreichtum, durch den sich R IV und R III auszeichnen.

Als besonders bemerkenswerte Form tritt innerhalb dieser xerotherm geprägten Schneckengesellschaften wieder die mediterrane Art Zebrina detrita auf, die schon in Abschnitt A2 des Komplexes A als Begleiter seiner im Artenbestand weitgehend übereinstimmenden, xerothermen Malakofauna nachgewiesen werden konnte. Die Problematik dieses Fundes wurde oben schon dargelegt. Die Anwesenheit dieser Art in zwei nebeneinanderliegenden und voneinander unabhängig zur Ablagerung gelangten altholozänen Sedimentkomplexen ist als Indiz dafür zu werten, daß Zebrina schon im Altholozän wenigstens vorübergehend Bestandteil der süddeutschen Molluskenfauna war, ehe sie dann im Jungholozän (möglicherweise in einer zweiten Einwanderungswelle) einen festen Platz in unserer Fauna einnehmen konnte.

Als weiteres mediterranes Faunenelement ist in R IV die Nadelschnecke Cecilioides acicula vertreten. Der Nachweis, daß diese Form schon im Altholozän Bestandteil der süddeutschen Schneckenfauna war, fällt wegen der unterirdischenLebensweise dieser Art, bei der sie bis $2 \mathrm{~m}$ tief in den Untergrund einzudringen vermag und so leicht in ältere Sedimente gelangen kann, einigermaßen schwer. So gibt es bis jetzt keine sicheren Angaben darüber, ab welcher Phase des Holozäns sie in Mitteleuropa fest Fuß gefaßt hat. Auch der vorliegende Fund läßt diese Frage unbeantwortet.

Neben den xero- und thermophilen Schnecken sind die wärme- und feuchtigkeitsliebenden Holozän-Arten in R IV und R III durch eine ganze Reihe von Formen vertreten (Carychium tridentatum, Acanthinula aculeata, Ena obscura, Discus rotundatus, Aegopinella pura, Aegopinella sp., Limax sp., Monachoides incarnata, Cepaea hortensis) und erreichen mit 8,3 bzw. 6,0\% der Individuen und 26,5 bzw. 25,8\% der Arten einen ähnlich starken Anteil an der Fauna wie in Abschnitt A2 des Fundkomplexes A (Abb. 6).

Bei einem Vergleich der Molluskenspektren der Komplexe A und R (Abb. 3-6) sind Beziehungen zwischen A2 und R IV bzw. R III unverkennbar. Es ist deshalb zu vermuten, daß es sich hier um Sedimente handelt, die ungefähr gleiches Alter haben und daß beide in einer Phase starker Erwärmung innerhalb des Altholozän zur Ablagerung kamen.

In R II und R I, den beiden obersten Abtragungen des Fundkomplexes R, zeichnen sich die Faunen durch besondere Arten- und Individuenarmut aus (Tabelle 6). Mit 55,1 \% (R II) und 72,7\% (R I) der Individuen erreicht hier Discus ruderatus einen Anteil an der Gesamtfauna, der die dominierende Stellung dieser Art in den übrigen Abtragungen des 
Komplexes $\mathrm{R}$ noch beträchtlich übertrifft. Auffallend schwach vertreten sind hier die xeround thermophilen Faunenelemente und auch die sonst so häufige Clausilia dubia. Hier macht sich offenbar das Auffüllen der Höhlenruine und die damit zusammenhängende zunehmende Einschränkung der freien Felsflächen, die die Wohnplätze dieser Formen bildeten, bemerkbar. Während der Anteil der xero- und thermophilen Arten in R III noch fast $20 \%$ der Individuenzahl betrug, sind sie in R II und R I nur mehr mit $7 \%$ bzw. 2,5\% der Individuen vertreten. Ausgesprochen südliche Arten fehlen in R II und R I vollständig.

Die wärme- und feuchtigkeitsliebenden Holozänarten sind zwar in R II und R I mit durchschnittlich 13,25\% der Individuen zahlenmäßig etwas stärker vertreten als in den übrigen Abtragungen des Komplexes $\mathrm{R}$ (Abb. 6). Sie werden jedoch nur durch einige wenige Arten (Aegopinella div. sp. Discus rotundatus und Limax sp.) repräsentiert, die darüberhinaus zu den anspruchslosesten dieser Gruppe gehören.

Die Schneckenfaunen der beiden obersten Abtragungen des Komplexes $\mathrm{R}$ lassen sich vielleicht am ehesten als verarmte und anspruchslose Waldfaunen bezeichnen, denen einige wenige, an Felsen siedelnde Formen des offenen Geländes beigemischt sind. Nach der auf Grund der Analyse der Gastropodenfaunen in R IV und R III anzunehmenden starken Erwärmung im Altholozän, weisen die Faunen aus R II und R I auf feuchtere und kühlere Umweltbedingungen in der Folgezeit hin.

\section{Holzkohlendiagramm}

\begin{tabular}{|c|c|c|c|c|c|c|c|}
\hline \multirow[t]{3}{*}{ Straten } & \multicolumn{6}{|c|}{ Hauptarten in: } & \multirow[b]{2}{*}{$\begin{array}{l}\text { indiffe- } \\
\text { rent }\end{array}$} \\
\hline & \multicolumn{4}{|c|}{ Eichenmischwald } & \multicolumn{2}{|c|}{ Föhrenwald } & \\
\hline & $\begin{array}{l}\frac{n}{\partial} \\
\frac{d}{\partial} \\
\text { d. } \\
50 \%\end{array}$ & 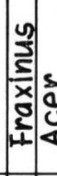 & $\frac{n}{3}$ & 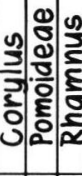 & $\stackrel{\text { Df }}{\text { D }}_{50 \%}$ & $\frac{\frac{0}{3}}{\frac{1}{0}}$ & 网 \\
\hline A1 & & & & & & $\bullet$ & $\bullet$ \\
\hline A2 & 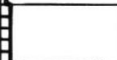 & $\bullet$ & $\bullet$ & $\bullet \bullet$ & WIII & & - \\
\hline RI-IV & & $\bullet$ & $\bullet$ & $\bullet \bullet \bullet$ & & & $\bullet$ \\
\hline RV+ZII & $\theta$ & & & $\bullet$ & Enta & & \\
\hline
\end{tabular}

Abb. 7. Euerwanger Bühl. Holzkohlendiagramm der Komplexe A und R von F. Schweingruber erstellt.

Die Analyse der Holzkohlenreste (Abb. 7) zeigt innerhalb der Abfolge R VI bis R I eine gewisse Gliederung. In R VI und R V ist Pinus mit mehr als 50\% vertreten. Der Rest wird bis auf geringe Reste von Pumoideae durch Quercus gebildet. In R IV bis R I tritt dagegen Pinus fast ganz zurück, während nun Quercus mehr als $80 \%$ erreicht. Daneben treten weitere Formen des Eichenmischwaldes, nämlich Fraxinus, Acer, Ulmus, Corylus und Rhamnus. Schweingruber stuft diesen oberen Profilabschnitt in das späte Boreal oder das Atlantikum ein. Es ist auffallend, daß sich die Komplexe R und A, die sich nach den zoologischen Befunden korrelieren ließen, eine so unterschiedliche Zusammensetzung in den Holzkohlen aufweisen. Das kann bedeuten, daß durch Zufälligkeiten in diesem Fall ein erheblicher Unterschied zwischen Vegetationsbild und Holzkohlenüberlieferung erscheint. Deswegen muß hier verzichtet werden, die vorliegenden Daten der Holzkohlenanalyse näher zu interpretieren. 


\section{Die Faunen des Euerwanger Bühls und ihre Beziehungen zu anderen süddeutschen Faunen aus dem Würm und Altholozän}

Die Faunenkomplexe des Euerwanger Bühl erlauben punktuelle Einblicke in die Faunenentwicklung im Jungpleistozän und besonders im Altholozän. Die Bedeutung der Aufsammlungen liegt in der Möglichkeit, Ergebnisse aus den Untersuchungen an Säugetieren, an Gastropoden, an Holzkohle und an menschlichen Kulturhinterlassenschaften auf dem Hintergrund absoluter Datierungen miteinander zu korrelieren.

\section{Das Jungpleistoz än}

Die Besonderheit der eindeutig kaltzeitlichen Gewöllefauna aus Komplex H liegt im Fehlen der beiden Lemminge (Dicrostonyx torquatus und Lemmus lemmus). Bevor die C14-Datierung vorlag, wurde diese Fauna versuchsweise mit einer spätpleistozänen Faunenassoziation aus der Petényi-Höhle im Bükk-Gebirge (NO-Ungarn) (Jánossy 1960) verglichen, wo während der beginnenden Wiedererwärmung in den Lagen 5-3 die Lemminge früher zurückgehen als die anderen kaltzeitlichen Arten. Zu dieser Einstufung paßte auch das - wenn auch äußerst spärliche - Auftreten von Clethrionomys im Euerwanger Bühl H. Die C14-Datierung verweist aber auf ein wesentlich höheres Alter dieser Fauna. Mit 26500 Jahren BP ist sie innerhalb des Würm-Glazials zwischen dem Denekamp-Interstadial (etwa 29000-32000 BP) und dem Tursac-Interstadial (etwa 22000 bis $23000 \mathrm{BP}$ ) anzusetzen. Es liegen zwar zahlreiche kaltzeitliche Kleinsäugerfaunen aus dem letzten Glazial in Süddeutschland vor, aber die feinstratigraphische Einstufung innerhalb der vielgliedrigen Abfolge von Stadialen und Interstadialen konnte fast nie erfolgen, so daß wir zur Zeit noch weit davon entfernt sind, eine detaillierte Kenntnis der Faunenentwicklung während des letzten Glazials zu haben. Das Mikrofaunenprofil aus den Weinberghöhlen bei Mauern zeigt deutliche langfristige Häufigkeitsschwankungen für die Lemminge (v. Koenigswald, Müller-Beck \& Pressmar 1974). Hier kann allerdings das Minimum von Dicrostonyx in Schicht D zeitlich mit dem Denekamp-Interstadial in Verbindung gebracht werden, da eine C14-Datierung mit $28265 \pm 325 \mathrm{BP}$ (Gr N - 6095) vorliegt. Damit ist diese Fauna älter als der Komplex $\mathrm{H}$ vom Euerwanger Bühl. Da es sich im Komplex $\mathrm{H}$ aber um eine unausgewogene Fauna handelt, der nicht nur die Lemminge, sondern auch alle Insektivoren fehlen, wollen wir diese Fauna weder zu ökologischen noch feinstratigraphischen Interpretationen heranziehen, ehe nicht weitere Faunen in diesen speziellen Zeitraum eingestuft werden können.

Zwischen dem kaltzeitlichen Komplex $\mathrm{H}$ und dem bereits altholozänen Komplex $\mathrm{F}$ liegt der Hochstand der Vereisung sowie das schrittweise Zurückweichen der Gletscher. Faunistisch ist die Zeit des Hochstandes der Gletscher nur ungenügend belegt. Erst mit dem Abklingen der Vereisung wird die Faunendokumentation wieder besser. Nur eine Phase der sicher mehrgliedrigen Faunenabfolge ist in der Kleinsäugerfauna hinreichend belegt. Es ist die bereits von KoKen 1909 beschriebene „obere Nagerschicht“. Diese Faunenassoziation ist durch eine Dominanz von Dicrostonyx mit etwa $50 \%$ der Nagetiere gekennzeichnet. KOKEN hat dieses Niveau außer am Sirgenstein auch an der Kleinen Scheuer am Hohlenstein im Lonetal untersucht. Nachgrabungen in der Kleinen Scheuer (Hahn \& Koenigswald im Druck) ermöglichten eine Einstufung dieser letzten Dominanz von Dicrostonyx in die älteste Dryaszeit durch eine C14-Datierung mit $13252 \pm 98 \mathrm{BP}$ (H 4183 - 3418). Nach dieser Phase geht der Anteil von Dicrostonyx schnell zurück und die holozänen Arten treten in den Vordergrund (v. Koenigswald \& Taute 1974).

\section{Das Altholoz än}

Über den Zeitpunkt, wann die holozän geprägte Fauna nach Süddeutschland eingewandert ist und die kaltzeitlichen Arten verdrängt hat, gibt es noch keine genauen Un- 
tersuchungen. In Analogie zu den Verhältnissen in Norddeutschland wurde angenommen, daß die warmzeitliche Fauna erst nach dem Ende der jüngeren Dryaszeit, dem letzten Kälterückschlag des Pleistozäns, aufgetreten ist. Nach der nordwesteuropäischen Gliederung (TAuber 1970) liegt das Ende der jüngeren Dryaszeit bei 10200 BP. In diesem Zusammenhang ist bedeutsam, daß in den Komplexen F, E und D vom Euerwanger Bühl, deren absolute Datierungen in die erste (ältere) Hälfte des 10. Jahrtausends BP verweisen (s. Abb. 8), der Faunenaustausch bereits abgeschlossen ist. Es wäre nun sicher voreilig, anzunehmen, daß sich die gesamte Umschichtung der Fauna in den dazwischen liegenden 500 Jahren vollzogen hätte. Diese Zeit erscheint für eine so wesentliche Umwandlung zu kurz. Daher ergibt sich die Frage, ob diese holozän geprägte Fauna nicht schon früher, etwa in der der jüngeren Dryaszeit vorausgehenden Wärmeschwankung, dem ebenfalls zum Pleistozän gehörenden Alleröd, eingewandert ist. Diese Frage kann zwar am Material des Euerwanger Bühls nicht entschieden werden, es gibt aber Argumente, die einen so

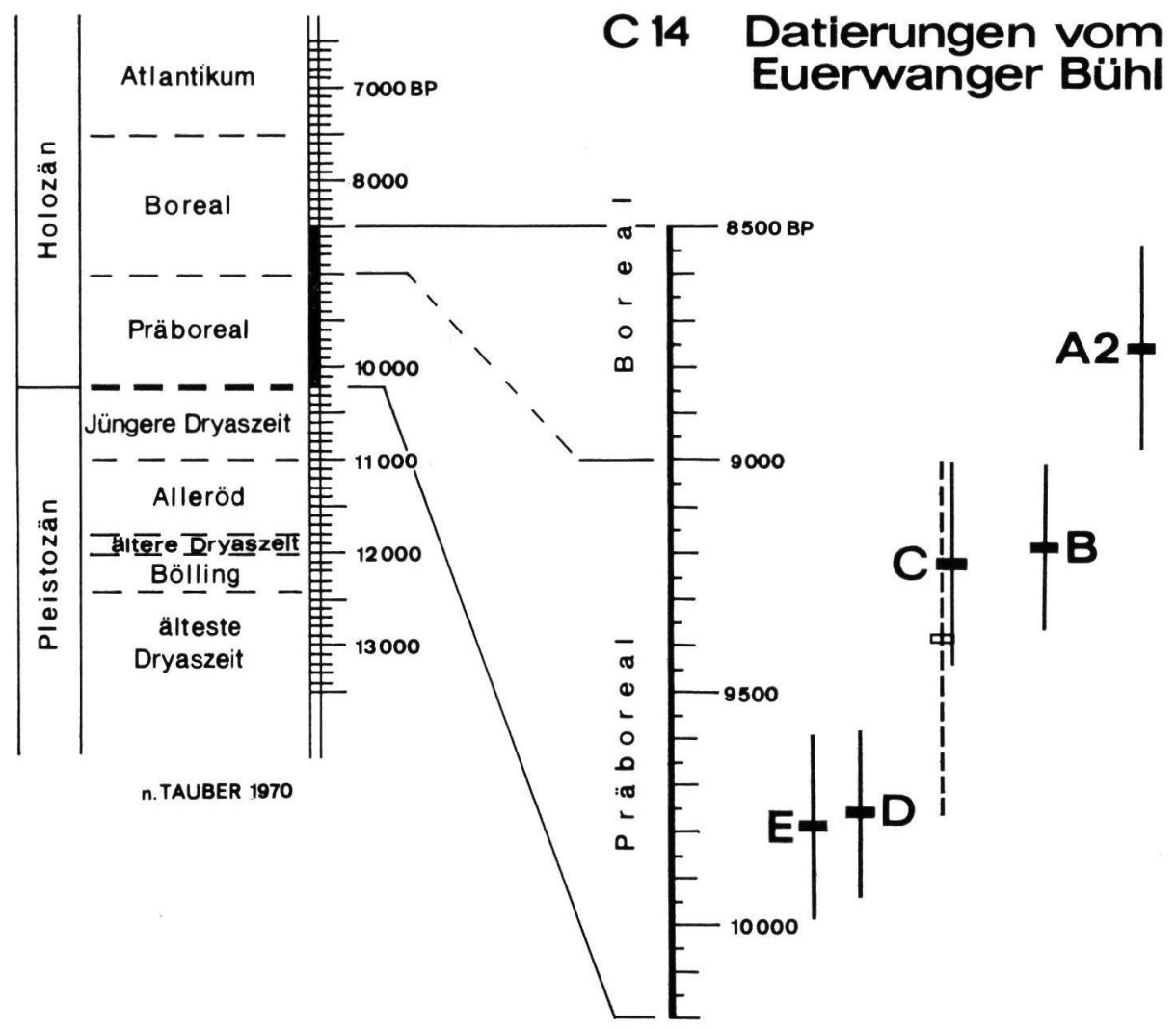

Abb. 8. Euerwanger Bühl. Die C14-Datierungen (Mittelwert und statistischer Fehler von $\pm 2 \sigma=95 \%$ Wahrscheinlichkeit) der altholozänen Faunenkomplexe und die Abgrenzung für die Pollenzonen des Spät- und Postglazials in Nordwesteuropa (nach TAuber 1970). Die Faunenkomplexe wurden durch Holzkohle im Institut für Umweltphysik der Universität Heidelberg datiert.
A 2 (H $4047-3192) 8760 \pm 110 \mathrm{BP}$
C $(\mathrm{H} 3605-2777) 9225 \pm 110 \mathrm{BP}$
B (H 3604-2751) $9190 \pm 90$
C (H 3605-2760) $9390 \pm 190$

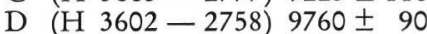
E (H 3603-2759) $9790 \pm 100$

Die nachträgliche Datierung des Komplexes $\mathrm{H}$ erfolgte mit Kollagen aus Kleinsäugerknochen (H 4278 - 3525) $26500 \pm 800$ BP. 
frühen Faunenaustausch wahrscheinlich machen. Das Alleröd konnte in den Faunen Süddeutschlands noch nirgendwo sicher erkannt werden, aus dem Rheinland aber, wo dieser Zeitabschnitt in Profilen durch die Einschaltung des Laacher Bimstuffes markiert ist, ist z. B. das Reh - eine der für holozäne Faunen typischen Formen - im Alleröd sicher belegt (z. B. in Ochtendung im Neuwieder Becken [BoekER et al. 1972]). Es besteht daher kein Grund daran zu zweifeln, daß das Reh zu dieser Zeit auch in Süddeutschland verbreitet war. Der dem Alleröd folgende Kälterückschlag (die jüngere Dryaszeit) ist zwar in Norddeutschland durch Rentierfaunen und den Rückgang der Waldvegetation gekennzeichnet, in den Pollenprofilen Süddeutschlands tritt diese Klimaverschlechterung außerhalb der Gebirge jedoch kaum in Erscheinung (GRONBACH 1961, GöTtLich 1960, LANG 1962). Daher erscheint es möglich, daß hier der Faunenaustausch bereits im Alleröd begonnen hat und durch die jüngere Dryaszeit - im Gegensatz zu Norddeutschland nicht mehr wesentlich beeinflußt worden ist. Unter diesen Voraussetzungen verwundert es nicht, daß im frühen Präboreal bereits eine reine Holozän-Fauna am Euerwanger Bühl belegt ist.

Die Funde vom Euerwanger Bühl erlauben es, besser als dies bisher möglich war, die Faunen des Holozäns von denen des späten Pleistozäns abzugrenzen und innerhalb des Holozäns jene des Altholozäns gegenüber jüngeren zu charakterisieren.

Die holozäne Kleinsäugerfauna unterscheidet sich in Süddeutschland von denen des Pleistozäns einerseits dadurch, daß Cletbrionomys und Apodemus einen sehr beträchtlichen Anteil an der Nagerfauna stellen. Die beiden Arten kommen in kaltzeitlichen Faunen nur sporadisch vor. Andererseits ist das Wiedereinwandern der Interglazialformen Crocidura und Sciurus vulgaris kennzeichnend für die Wiedererwärmung nach dem letzten Glazial. Für die älteren Abschnitte innerhalb des Holozäns sind einige Arten kennzeichnend, die heute in der süddeutschen Fauna überhaupt fehlen oder nur eine sehr untergeordnete Rolle spielen. Es sind vor allem: Pitymys subterraneus, Microtus oeconomus, Sicista betulina und Cricetus. Während Pitymys subterraneus sich erst im Postglazial ausgebreitet hat, sind die anderen Arten bereits Bestandteile der kaltzeitlichen Kleinsäugerfauna. Pitymys ist in den altholozänen Faunen mit großer Regelmäßigkeit anzutreffen. Am Euerwanger Bühl ist diese Art in fast jedem Komplex belegt. Auch in Ungarn konnte Jánossy 1960 ein Vordringen von Pitymys im Altholozän nachweisen. Rezent ist Pitymys in Süddeutschland nur sporadisch vertreten.

Von den genannten Arten ist Cricetus im Komplex B und damit für das Präboreal belegt. Ein weiterer Beleg für das Altholozän in Bayern stammt aus der Spaltenfüllung Belzheim im Ries (Bolten 1971). Im Mesolithikum ist Cricetus in Süddeutschland und in der Schweiz mehrfach nachgewiesen worden (z. B. Toвien 1938/39, Schmid 1964). Diese Ausbreitung hat auch Ostfrankreich eingeschlossen (CHAline 1973). Die Einführung des Ackerbaues im Neolithikum hat durch die Schaffung von Kultursteppen eine erneute Ausbreitung des Hamsters begünstigt, nachdem sich diese Art - wahrscheinlich durch die immer dichter werdende Bewaldung - zunächst zurückgezogen hatte. Die heutige Verbreitung umfaßt in Süddeutschland und besonders in der Schweiz keineswegs mehr das im Altholozän eingenommene Areal. Recht regelmäßig wird auch Microtus oeconomus, die nordische Sumpfmaus, in den Faunen des Altholozäns in Süddeutschland angetroffen. Allerdings ist ihr quantitativer Anteil an der Nagerfauna wesentlich geringer als in den kaltzeitlichen Faunen. Daß diese Art am Euerwanger Bühl fehlt, erklärt sich aus der lokalen Höhenlage der Fundstelle in einem Karstgebiet, das zumindest während gemäßigter Klima-Abschnitte recht trocken ist. Hier dürfte die feuchtigkeitsliebende Sumpfmaus kaum einen geeigneten Biotop gefunden haben. Diese Art kommt heute in Süddeutschland nicht mehr vor. Schließlich ist noch Sicista betulina, die Birkenmaus, als Bestandteil altholozäner Faunen zu nennen. Daß diese Art so selten gefunden wurde, hat wahrscheinlich 

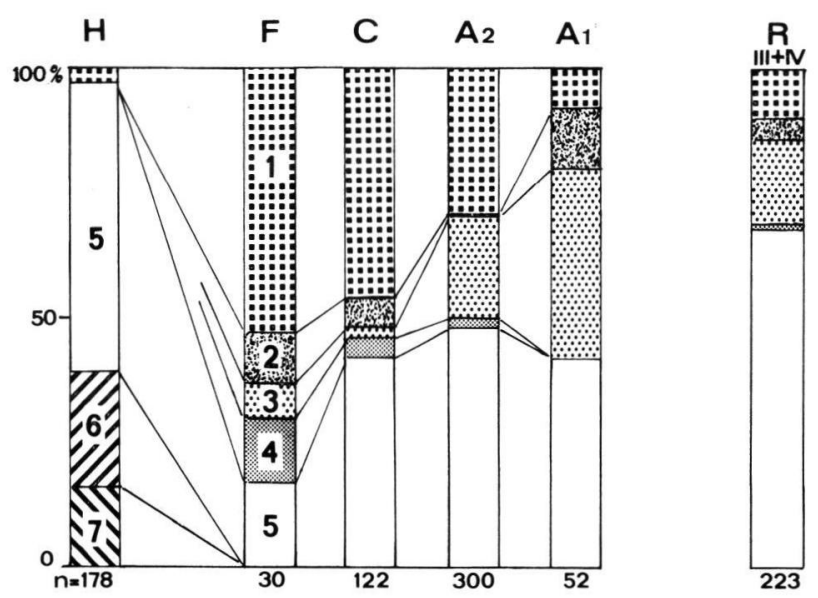

Abb. 9. Euerwanger Bühl. Klimabedingte Verschiebung in den prozentualen Anteilen der mittelgroßen Nager in den Komplexen H (Jungpleistozän), sowie F, C, A 2, A 1 und R (Altholozän).

$\begin{array}{ll}1 & \text { Clethrionomys glareolus } \\ 2 & \text { Pitymys subterraneus } \\ 3 & \text { Apodemus flavicollis } \\ 4 & \text { Apodemus sylvaticus }\end{array}$

5 Microtus arvalis-agrestis

6 Microtus gregalis

7 Microtus oeconomus

sammeltechnische Gründe. In der Burghöhle von Dietfurt bei Sigmaringen konnte genügend Material der altholozänen Schicht 12 a geschlämmt werden, und die Ausbeute ergab zahlreiche Sicista-Reste. Der genaue Zeitpunkt, wann sich das Verbreitungsgebiet dieser Arten reduziert hat, ist bisher sehr ungenügend bekannt, da bei den archäologischen Grabungen, die jüngere Perioden als das Mesolithikum betreffen, auf Kleinsäuger nur unzureichend geachtet wird. Ebenso ist der Zeitpunkt der Ausbreitung gewisser Arten als Kulturnachfolger (z. B. Epimys rattus, Mus musculus und Cricetus) nicht detailiert bekannt. Das Verschwinden der obengenannten Arten steht möglicherweise mit der starken Zunahme der Bewaldung im Atlantikum in Verbindung.

Untergeordnete Klimaschwankungen, die keinen Faunenaustausch bewirken, machen sich in der Kleinsäugerfauna nur dort bemerkbar, wo sich wichtige Verbreitungsgrenzen über das Gebiet verschieben. So zeichnet sich das Atlantikum in Norddeutschland durch das kurzfristige Auftreten zahlreicher südlicher Arten, in der Kleinfauna besonders Crocidura, ab (z. B. in Pisede; Peters, Heinrich, Beurton \& Jäger 1972). In Süddeutschland erweitert diese Klimaverbesserung den Faunenbestand nicht und läßt sich daher weniger leicht nachweisen.

Die holozäne Faunenfolge vom Euerwanger Bühl zeigt in der Kleinsäugerfauna keine tiefgreifende Veränderung. Es zeigt sich zwar eine Dominanz von Cletbrionomys in den älteren Komplexen (Abb. 9), die langsam - eventuell entsprechend der zunehmenden Bewaldung - abgebaut wird, aber diese Änderungen sind nicht sehr auffällig und bisher noch nicht durch weitere Profile als allgemeiner Entwicklungstrend gesichert.

Dieser sehr einheitlichen Säugetierfauna steht in der Gastropodenfauna eine sehr deutliche Verschiebung zwischen den Komplexen $\mathrm{R}$ und A2 einerseits und A1 andererseits gegenüber. In $\mathrm{R}$ und A2 liegen typische Discus ruderatus-Faunen vor, wie sie in Süddeutschland recht verbreitet sind (Dенм 1967, eine Ergänzung BolTEN 1971). Besonders bemerkenswert erscheint in diesem Zusammenhang die Vergesellschaftung der beiden sibiro-asiatischen Waldarten Discus ruderatus und Nesovitrea petronella. Ein solches ge- 
meinsames Vorkommen der beiden Arten ist für eine Reihe bisher bekannter süddeutscher Discus ruderatus- Faunen belegt (Huckriede 1965, Deнм 1967). Neben Discus ruderatus ist Nesovitrea petronella offenbar eine zweite, im Altholozän Süddeutschlands noch allgemein verbreitete Art, die sich erst infolge einer einschneidenden Klimaveränderung im mittleren Holozän aus den Niederungen in die kühleren Gebirgslagen zurückgezogen hat.

Möglicherweise ist in A1 ein Zurückgehen der altholozänen Discus ruderatus-Fauna belegt. Da A1 jedoch aus verschiedenen Gründen, die oben dargelegt worden sind, nicht sehr viel jünger sein dürfte als A2, scheint diese Faunenentwicklung schon vor dem Atlantikum eingesetzt zu haben, also früher, als das bisher (LožEK 1964, ANT 1965) angenommen wurde. Allerdings ist es bei Gastropodenfaunen, die stets sehr lokale Verhältnisse widerspiegeln, immer recht problematisch, von einer Fundstelle auf allgemeine stratigraphische Veränderungen zu schließen.

Wenn durch die Fauna vom Euerwanger Bühl auch eine Reihe neuer Fragen aufgeworfen wurden, die sich anhand des vorliegenden Materials nicht ausreichend beantworten lassen, so hat dieser Fundplatz dennoch wesentliche Erkenntnisse über die Entwicklung der Kleinsäuger- und Schneckenfauna im Jungpleistozän und vor allem im Altholozän geliefert.

\section{Literatur}

ANT, H.: Der boreoalpine Verbreitungstypus bei europäischen Landgastropoden. - Zool. Anz. Suppl. 28, 326-335. Leipzig 1965.

Boeker, M., Lehmann, E. von \& Remy, H.: Über eine Wirbeltierfauna aus den jüngsten würmzeitlichen Ablagerungen am Michelberg bei Ochtendung/Neuwieder Becken. - Decheniana 124, 119-134, Bonn 1972.

Bolten, R.: Eine postglaziale Karstfüllung bei Belzheim/Ries (Bayern). — Mitt. Bayer. Staatssamml. Paläont. hist. Geol. 11, 329-332, 1 Abb., München 1971.

Chaline, J.: Biogéographie et fluctuations climatique au Quaternaire d'après les faunes de rongeurs. - Acta Zool. Cracoviensia 28, 7, Krakau 1973.

Deнм, R.: Die Landschnecke Discus ruderatus im Postglazial Süddeutschlands. - Mitt. Bayer. Staatssamml. Paläont. hist. Geol. 7, 135-155. München 1967.

Gaffrey, G.: Die Schädel der mitteleuropäischen Säuger. - Abh. Ber. Staatl. Mus. Tierk. Forschinst. Dresden 21, 5-123, 75 Abb., Leipzig 1953.

Götтlich, K.: Beiträge zur Entwicklungsgeschichte der Moore in Oberschwaben. - Jh. Ver. vaterl. Naturkde. Württ. 115, 93-174, 24 Abb., Stuttgart 1960.

Gronbach, G.: Pollenanalytische Untersuchungen zur Geschichte des Federsees und zur vorgeschichtlichen Besiedlung des Federseerieds. - In: Zimmermann, W., Der Federsee, 316-355, Stuttgart 1961.

Haesslein, L.: Die Mollusken der Falkensteingrabung von Tiergarten (Schwäbische Alb). - Jh. Ver. vaterl. Naturkde. Württ. 107, 60—71, Stuttgart 1952.

Hahn, J. \& Koenigswald, W. v.: Die steinzeitlichen Funde und die spätglaziale Nagetierschicht in der Kleinen Scheuer am Hohlenstein im Lonetal. - Fundberichte aus Baden-Württemberg 3, Stuttgart (im Druck).

Heller, F.: Zwei Pferdeunterkiefer aus dem Pleistozän der südlichen Frankenalb. - Quartär 23/24, 165-173, 1 Taf., Bonn 1973.

Huckriede, R.: Eine frühholozäne ruderatus-Fauna im Amöneburger Becken. (Mollusca, Hessen). — Notizbl. hess. L. A. Bodenforschung 93, 196-206, Taf. 12, Wiesbaden 1965.

JáNossy, D.: Neuere Angaben zur Kenntnis der postglazialen und holozänen Kleinvertebratenfauna Ungarns. - Ann. hist. natur. Mus. nat. Hungarici 51, 113-119, 1 Abb., Budapest 1959.

- : Nacheiszeitliche Wandlung der Kleinsäugerfauna Ungarns. - Zool. Anz. 164, 114-121, 2 Abb., Leipzig 1960.

JÁNossr, D. \& Schmidr, E.: Die Nahrung des Uhus (Bubo bubo). Regionale und erdzeitliche Änderungen. - Bonn. zool. Beitr. 21, 25-51, Bonn 1970. 
Koenigswald, W. v.: Der Faunenwandel an der Pleistozän-Holozän-Grenze in der steinzeitlichen Schichtenfolge vom Zigeunerfels bei Sigmaringen. (Vorber.) - Archäol. Inf. 1, 41-45, $1 \mathrm{Abb}$., Tübingen 1973.

Koenigswald, W. v., Müller-Beck, H. \& Pressmar, E.: Die Archäologie und Paläontologie in den Weinberghöhlen bei Mauern (Bayern). - Archaeologica Venatoria 3, Tübingen 1974.

Koenigswald, W. v. \& TAute, W.: Mensch und Fauna unter dem Einfluß des Klimawandels an der Grenze vom Pleistozän zum Holozän. - Nachr. Deutsch. Geol. Ges. 9, 145-150, Hannover 1974.

Koken, E.: Diluvialstudien. - N. Jb. Min. Geol. Paläont. 1909, 57-90, 3 Taf., 12 Abb., Stuttgart 1909.

Kumerloeve, H.: Gewöllstudien an einem Sumpfohreulen-Brutpaar auf der Insel Amrum. Ornithol. Mitt. 20, 33-34, Stuttgart-Hamburg 1968.

LANG, G.: Zur späteiszeitlichen Vegetations- und Florengeschichte Südwestdeutschlands. - Flora 139, 243-294, Jena 1952.

LožEK, V.: Quartärmollusken der Tschechoslowakei. - Rozpravy Ústr. úst. geol. 31, 373 S., 91 Abb., 32 Taf., Prag 1964.

MARKert, D.: Schlüssel zur Bestimmung süddeutscher Ophidier-Wirbel und dessen Anwendung auf altholozänes Reptil-Material aus dem Euerwanger-Bühl (Franken). - N. Jb. Geol. Paläont. Abh. 149, 211-216, 6 Abb., Stuttgart 1975.

Peters, G., Heinrich, W.-D., Buerton, P. \& Jüger, K.-D.: Fossile und rezente Dachsbauten mit Massenanreicherungen von Wirbeltieren. - Mitt. Zoo. Mus. Berlin 48, 415-435, 9 Abb., Berlin 1972.

Schmid, E.: Die Tierknochen. - In: Band, H.-G.: Birsmatten-Basisgrotte. Eine mittelsteinzeitliche Fundstelle im unteren Birstal. - Acta Bernensia 1, 93-100, Bern 1964.

Sтовсн, G.: Jungpleistozäne Kleinsäugerfunde (Mammalia: Insectivora, Chiroptera, Rodentia) aus der Brillenhöhle. - In: RIEK, G.: Das Paläolithikum der Brillenhöhle bei Blaubeuren (Schwäbische Alb). - Forsch. Ber. Vor- u. Frühgesch. Baden-Württemberg 4, Stuttgart 1973.

— : Die Pleistozän-Holozän-Grenze bei Arvicola in Süddeutschland. - Symposium Therilogicum II, (Brno 1971), 347-353, Prag 1974.

TAUBER, H.: The Scandinavian varve Chronology and C14-Dating. - 173-196, 4 Abb. In: Olsson, I. U.: Radiocarbon Variations and absolute Chronology. - Nobel Symposium 12, Stockholm 1970.

TAUTE, W.: Untersuchungen zum Mesolithikum und zum Spätpaläolithikum im südlichen Mitteleuropa. - Band 1: Chronologie Süddeutschlands. - Habilitationsschrift, Tübingen 1971 (Manuskript).

ToBien, H.: Die Kleinsäugerreste aus der Falkensteinhöhle im oberen Donautal. - Bad. Geol. Abh. 10, 126-130, Karlsruhe 1938/39.

Manuskript eingeg. 17. 4. 1975.

Anschriften der Verfasser: Dr. Wighart v. Koenigswald, Geol.-Paläont. Institut der Universität Tübingen, 74 Tübingen 1, Sigwartstr. 10; Dr. Wolfgang Rähle, Institut für Biologie III der Universität Tübingen, Lehrstuhl Zoologie, 74 Tübingen, Auf der Morgenstelle 28. 